\title{
Can string theory predict the Weinberg angle?
}

\author{
Stuart Raby and Akın Wingerter \\ Department of Physics, The Ohio State University, 191 W. Woodruff Ave, Columbus, Ohio 43210, USA
}

(Received 12 June 2007; published 26 October 2007)

\begin{abstract}
We investigate whether the hypercharge assignments in the standard model can be interpreted as a hint at grand unification in the context of heterotic string theory. Our analysis is a follow-up to the recent minilandscape analysis of the $\mathbb{Z}_{6}$-II orbifold of the $\mathrm{E}_{8} \times \mathrm{E}_{8}^{\prime}$ heterotic string. In that analysis, an intermediate grand unified theory (GUT) was a requirement for finding MSSM-like theories. Nevertheless, about $1 \%$ of the models in this mini-landscape were MSSM-like. In this paper we remove this GUT restriction. To this end, we introduce a general method to calculate $\mathrm{U}(1)_{Y}$ for any heterotic orbifold and compare our findings to the cases where hypercharge arises from a GUT. Surprisingly, in the overwhelming majority of 3-2 standard models, a nonanomalous hypercharge direction can be defined, for which the spectrum is vectorlike. For these models, we calculate $\sin ^{2} \theta_{w}$ to see how well it agrees with the standard GUT value. We find that $12 \%$ have $\sin ^{2} \theta_{w}=3 / 8$, while all others have values which are less. Finally, $89 \%$ of the models with $\sin ^{2} \theta_{w}=3 / 8$ have $\mathrm{U}(1)_{Y} \subset \mathrm{SU}(5)$.
\end{abstract}

DOI: 10.1103/PhysRevD.76.086006

PACS numbers: $11.25 . \mathrm{Mj}$

\section{INTRODUCTION}

In a recent paper, Lebedev et al. [1] performed a "minilandscape" search in the heterotic string looking for MSSM-like models. The search focused on the $\mathbb{Z}_{6}$-II orbifold and quite dramatically it was found that about 1 in 300 theories were MSSM-like. Why should there be such a "fertile patch" in the string landscape can be understood in terms of the property of orbifold GUTs and "local GUTs" as emphasized in the following Refs. [2-8]. In the $\mathbb{Z}_{6}$-II orbifold of the heterotic string, four particular shift vectors were identified that break the visible $\mathrm{E}_{8}$ gauge symmetry to $\mathrm{E}_{6}$ or $\mathrm{SO}(10)$. Then in the first twisted sector (see Fig. 1) it was shown that massless chiral multiplets in the 27 of $\mathrm{E}_{6}$ or $\mathbf{1 6}$ of SO(10) exist, residing at "local" GUT twisted sector fixed points. In fact, with one Wilson line lying in the $\mathrm{SO}(4)$ torus, there are exactly two possible families of quarks and leptons. With two Wilson lines, it was possible to break the visible $\mathrm{E}_{8}$ to the standard model $(\mathrm{SU}(3) \times$ $\mathrm{SU}(2) \times \mathrm{U}(1)_{Y} \times$ a hidden sector gauge symmetry). Two families were localized at the local GUT fixed points and the third family and Higgs doublets were found in a combination of the untwisted and twisted sectors. In the "mini-landscape" search [1], the SM (including hypercharge) was constrained to lie within $\mathrm{SU}(5) \subset \mathrm{SO}(10)$ or $\mathrm{E}_{6}$. This short review describes the "fertile patch" in the landscape and hopefully makes it clear why it was so fertile.

In the present paper we also focus on the $\mathbb{Z}_{6}$-II orbifold with the same shifts. We inherit the same $\mathrm{SO}(10)$ symmetry at the two $\mathrm{SO}(4)$ fixed points. Our search differs from Ref. [1] in that we do not demand that the SM sit within $\mathrm{SU}(5) \subset \mathrm{SO}(10)$. Our search is more general, so that the fundamental question we address is - to what extent do MSSM-like models require an intermediate GUT? In the following Sec. II we refine this question. In Sec. III we outline our more general search for MSSM-like models.
Then in Sec. IV we discuss our results, including a comparison to the mini-landscape analysis.

\section{HYPERCHARGE FROM GRAND UNIFICATION}

In string compactifications aiming at the standard model in four dimensions, one is typically left with a large number of Abelian factors, ${ }^{1}$

$$
\mathrm{SU}(3) \times \mathrm{SU}(2) \times \mathrm{U}(1)^{n} \times \text { non-Abelian factors. }
$$

A priori, hypercharge may be any linear combination of the $\mathrm{U}(1)$ 's that gives the correct values on the elementary particles, cf. Table I. What complicates matters even more is the fact that generically, the spectrum contains a large number of representations that may play the roles of quarks, leptons, and Higgses in the low-energy theory. In order not to be in gross contradiction to experimental observation, the spectrum is required to be vectorlike so that the exotic particles ${ }^{2}$ may decouple by acquiring a large Majorana-type mass. In this context, it is not clear which representations are to be identified with families or exotics, respectively. As a result, we are left with a large number of choices, which makes finding a U(1) which may play the role of hypercharge a hard problem to solve.

The problem is not so difficult to solve, if we assume some additional structure. Grand unification [11-13] offers a default choice for hypercharge. In Georgi-Glashow $\mathrm{SU}(5)$ [11], $\mathrm{U}(1)_{Y}$ is obtained as the Abelian factor that is left over after breaking SU(5) to the standard model

\footnotetext{
${ }^{1}$ In (heterotic) orbifold constructions, U(1) factors may be broken by the mechanism of continuous Wilson lines, as explored in Refs. [9,10].

${ }^{2}$ All the additional particles that are not in the MSSM are termed exotics. If the exotics are such that they can be combined to pairs of representations that are conjugates of each other, the spectrum is called vectorlike.
} 

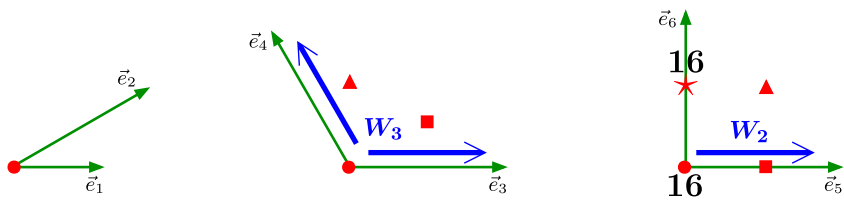

FIG. 1 (color online). The first twisted sector of the $\mathbb{Z}_{6}$-II orbifold. The shift vectors of the mini-landscape search preserve a local $\mathrm{SO}(10)$ (or $\mathrm{E}_{6}$ ) GUT at the fixed points $(\bullet \bullet \bullet$ ) and $(\bullet \star$ ) with two 16 (or 27) dimensional massless multiplets.

TABLE I. Matter content of the standard model. In our conventions, $Q=T_{3 L}+\frac{Y}{2}$.

\begin{tabular}{llllll}
\hline \hline$Q$ & $(\mathbf{3}, \mathbf{2})_{1 / 3}$ & $L$ & $(\mathbf{1}, \mathbf{2})_{-1}$ & $H$ & $(\mathbf{1}, \mathbf{2})_{1}$ \\
\hline $\bar{u}$ & $(\overline{\mathbf{3}}, \mathbf{1})_{-4 / 3}$ & $\bar{e}$ & $(\mathbf{1}, \mathbf{1})_{2}$ & $\bar{H}$ & $(\mathbf{1}, \mathbf{2})_{-1}$ \\
$\bar{d}$ & $(\overline{\mathbf{3}}, \mathbf{1})_{2 / 3}$ & $\bar{\nu}$ & $(\mathbf{1}, \mathbf{1})_{0}$ & & \\
\hline \hline
\end{tabular}

gauge group, see Fig. 2. More specifically, the hypercharge direction [14] is given by the dual of the root that is projected out:

$$
\begin{aligned}
Y_{\mathrm{GG}} & =\frac{5}{3} \alpha_{3}^{*}=\sum_{j} \frac{5}{3}\left(A_{\mathrm{SU}(5)}^{-1}\right)_{3 j} \alpha_{j} \\
& =\frac{1}{3}\left(2 \alpha_{1}+4 \alpha_{2}+6 \alpha_{3}+3 \alpha_{4}\right)
\end{aligned}
$$

This works not only in the case where we start with a grand unified gauge group in four dimensions, but also in any other construction where the standard model is realized as a subset of a grand unified group. A particular example of this type is a field theoretic or stringy orbifold where grand unification is realized in higher dimensions $[3,15]$.

In a recent publication [1], we exploited this very fact ${ }^{3}$ to derive 223 heterotic $\mathbb{Z}_{6}$-II orbifold models with three generations of quarks and leptons, one pair of Higgses, and a vectorlike spectrum (see Fig. 3). The first step in the analysis was a judicious choice of a shift vector leading to either $\mathrm{SO}(10)$ or $\mathrm{E}_{6}$, with the additional requirement that at least one family representation, $\mathbf{1 6}$ or 27, respectively, be localized in the first twisted sector. Wilson lines subsequently broke the gauge symmetry to that of the standard model and hypercharge was realized as the $\mathrm{U}(1)$ which lies in $\mathrm{SU}(5)$, cf. again Fig. 2.

In light of the success of this approach, one question immediately arises: How crucial was the assumption that the standard model gauge group be realized as a subgroup of $\mathrm{SO}(10)$ ? If we drop this assumption, can we still find a

\footnotetext{
${ }^{3}$ The SO(10) structure was required not only for hypercharge, but also for a couple of other reasons, such as gauge and Yukawa coupling unification, the presence of a $B-L$ symmetry, a discrete subgroup of which may be a candidate for $R$-parity, etc. A nice discussion of the role of $\mathrm{SO}(10)$ in the context of theories beyond the standard model can be found in Refs. [16,17].
}

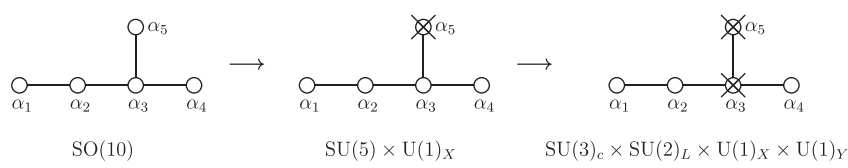

FIG. 2. From $\mathrm{SO}(10)$ to (un)flipped $\mathrm{SU}(5)$ to the standard model.

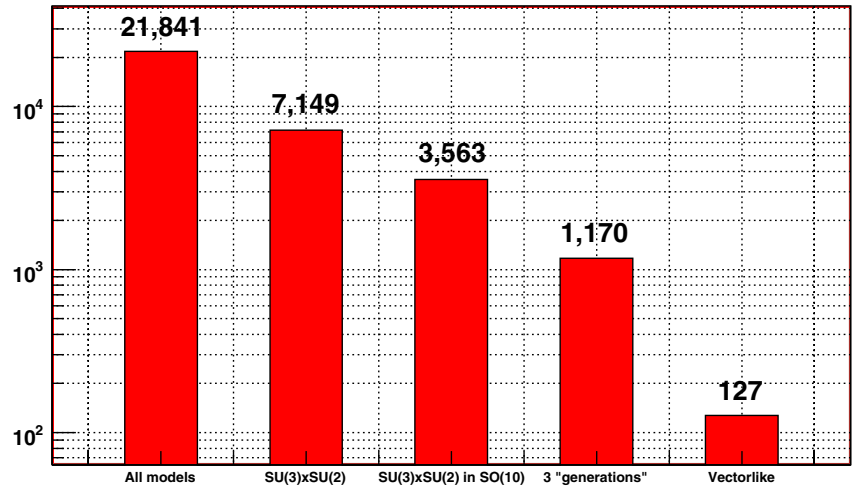

FIG. 3 (color online). Consider shift $V^{\mathrm{SO}(10), 1}$ of Ref. [1]. Of the (i) 21841 models, (ii) $33 \%$ contain the SM gauge group in at least one $\mathrm{E}_{8}$, (iii) $16 \%$ contain the SM gauge group as a subset of $\mathrm{SO}(10)$, (iv) $5 \%$ are three-generation standard models (w.r.t. color and weak isospin) lying in $\mathrm{SO}(10)$, and (v) $0.6 \%$ are three-generation standard models (now including hypercharge) with a vectorlike spectrum where hypercharge is that of GeorgiGlashow $\mathrm{SU}(5) \subset \mathrm{SO}(10)$.

suitable $\mathrm{U}(1)$ direction that plays the role of hypercharge such that all family and Higgs representations have the standard assignments and the remaining particles can be combined to vectorlike pairs that decouple from the lowenergy theory?

In Sec. III, we present a general method for constructing a suitable hypercharge out of any number of U(1) factors. Although we specialize to a heterotic orbifold model, it should be clear that the outlined methods are applicable in a much broader context.

\section{SEARCHING FOR HYPERCHARGE IN THE GENERAL CASE}

For the sake of clarity, we will consider a specific example along with the general construction. Table II shows the spectrum of a typical $\mathbb{Z}_{6}$-II orbifold model (More details are given in Appendix A).

In the present case, hypercharge may be any linear combination of 9 Abelian factors,

TABLE II. Three-generation model with gauge group $\mathrm{SU}(3) \times$ $\mathrm{SU}(2) \times \mathrm{U}(1)^{5} \times \mathrm{SU}(5)^{\prime} \times \mathrm{U}(1)^{14}$.

\begin{tabular}{cccc}
\hline \hline $3 \times(\mathbf{3}, \mathbf{2}, \mathbf{1})$ & $12 \times(\overline{\mathbf{3}}, \mathbf{1}, \mathbf{1})$ & $29 \times(\mathbf{1}, \mathbf{2}, \mathbf{1})$ & $8 \times(\mathbf{1}, \mathbf{1}, \mathbf{5})$ \\
\hline $6 \times(\mathbf{3}, \mathbf{1}, \mathbf{1})$ & $136 \times(\mathbf{1}, \mathbf{1}, \mathbf{1})$ & $8 \times(\mathbf{1}, \mathbf{1}, \overline{\mathbf{5}})$ \\
\hline
\end{tabular}




$$
\mathrm{U}(1)_{Y}=x_{1} \mathrm{U}(1)_{1}+x_{2} \mathrm{U}(1)_{2}+\ldots+x_{9} \mathrm{U}(1)_{9} \text {. }
$$

The naive ansatz of choosing a subset of the representations in Table II to be the observed elementary particles and then solving the linear equations for their having the correct hypercharges is not feasible. The number of such choices ${ }^{4}$ is at least

$$
\underset{>}{\left(\begin{array}{l}
3 \\
3
\end{array}\right) \times\left(\begin{array}{c}
12 \\
6
\end{array}\right) \times\left(\begin{array}{l}
6 \\
3
\end{array}\right) \times\left(\begin{array}{c}
29 \\
5
\end{array}\right) \times\left(\begin{array}{l}
5 \\
4
\end{array}\right) \times\left(\begin{array}{c}
216 \\
3
\end{array}\right) \times\left(\begin{array}{c}
216 \\
3
\end{array}\right)}
$$

corresponding to choosing (i) left-handed quark doublets, (ii) right-handed quarks, (iii) distinguishing up- and downtype quarks, (iv) 3 lepton doublets and 2 Higgses, (v) distinguishing leptons and up-type Higgs, (vi) righthanded electrons, (vii) right-handed neutrinos. (Note that in the above counting, the charges under the hidden sector gauge group are multiplicities for the standard model particles.)

Even if we were able to cope with such a large number of cases, the solutions to the linear equations will in general not give a vectorlike spectrum. From these considerations it should be clear that another line of thought has to be pursued.

In the remainder of this section, we describe our strategy for finding a suitable hypercharge even under the most unfavorable circumstances. For facility of inspection, in the following we will enumerate the key ideas entering our calculations.

\section{A. Exploiting the linear constraints}

(i) A judicious choice for the basis of U(1) directions greatly simplifies the calculations. In principle, one may choose any $n$ linearly independent directions that are orthogonal to the simple roots of the unbroken gauge group. However, from experience with grand unified theories we expect the $\mathrm{U}(1)$ directions (up to some multiplicative factor) to correspond to the duals of the roots that are projected out, so we can take them to lie in the root lattice ${ }^{5}$ of $\mathrm{E}_{8} \times \mathrm{E}_{8}^{\prime}$. This is not only, in some sense, the default choice, but it also then guarantees that $\mathrm{N}$-times the $\mathrm{U}(1)$ directions will have integer values ${ }^{6}$ on the representations, where we denote by $N$ the order of the orbifold.

\footnotetext{
${ }^{4}$ The model under consideration is relatively well-behaved with regard to the multiplicities of particles. There exist cases in which the net number of generations is realized by differences of larger multiplicities, thus considerably increasing the complexity of the problem.

${ }^{5}$ Observe that the root lattice of $\mathrm{E}_{8} \times \mathrm{E}_{8}^{\prime}$ is self-dual.

${ }^{6}$ The highest weight of a representation is of the general form $p+k V$, where $p$ is in the $\mathrm{E}_{8} \times \mathrm{E}_{8}^{\prime}$ root lattice, $k$ the number of the twisted sector, and $V$ the shift vector. Our assertion follows from the fact that $N V$ is in the root lattice.
}

(ii) Unfortunately, this assumption is not fully justified. Although it is generally true that we may always find $n$ linearly independent basis vectors that span the vector space orthogonal to the simple roots and at the same time belong to the $\mathrm{E}_{8} \times \mathrm{E}_{8}^{\prime}$ lattice, it is a priori not clear why hypercharge should lie in the lattice, or in other words, why the coefficients in Eq. (6) below should be integers. (The deeper reason behind this is the existence of chiral exotics which may have hypercharge assignments that are not multiples of $1 / 3$.) Therefore, we will drop the assumption on the coefficients when we consider the general case in Sec. III B.

(iii) Since assigning the correct hypercharges to all family representations has proved to be impractical, we make a compromise and demand only that the lefthanded quark doublets and the right-handed quark singlets have the correct values. In this case, there is still a choice to make, but the involved numbers are smaller by orders of magnitude. In the present example, this number ${ }^{7}$ is

$$
\left(\begin{array}{l}
3 \\
3
\end{array}\right) \times\left(\begin{array}{c}
12 \\
6
\end{array}\right) \times\left(\begin{array}{l}
6 \\
3
\end{array}\right)=18480
$$

Denoting the directions corresponding to the Abelian factors by capital letters, our ansatz takes the form

$$
Y=x_{1} U_{1}+x_{2} U_{2}+\ldots+x_{n} U_{n} .
$$

The hypercharge of a particular representation is given by the scalar product of its highest weight and $Y$. If we denote the highest weights of the lefthanded quark doublets and of the right-handed quark singlets by $\Lambda_{i}, i=1, \ldots, 9$, we obtain

$$
\Lambda_{i} \cdot Y=x_{1} \Lambda_{i} \cdot U_{1}+x_{2} \Lambda_{i} \cdot U_{2}+\ldots+x_{n} \Lambda_{i} \cdot U_{n} \text {, }
$$

which is a system of linear diophantine equations, provided we multiply the left- and right-hand-sides by $3 N$. We will henceforth not explicitly mention that the preceding and all following equations are to be multiplied by $3 N$. The factor of $N$ was explained in a footnote in Sec. III A, and the factor of 3 is the smallest common multiple of the denominators of the hypercharge assignments. Now it becomes very clear that in taking these equations to be diophantine, we restrict our search to such exotics which have hypercharge values with a denominator not greater than 3. We will lift this constraint in Sec. III B.

Although one may think that in our example, where we have 9 independent $\mathrm{U}(1)$ directions, these 9

\footnotetext{
${ }^{7}$ Despite this number being not so small, we find a solution in less than $1 \mathrm{~s}$ on a $2.7 \mathrm{GHz}$ Pentium computer. This means that one of the very first choices for the quarks allows for a hypercharge direction. Our experience shows that this is a general pattern.
} 
equations severely constrain the values of $x_{i}$, this is not true. In general, the system will be underdetermined, and, as a matter of fact, in our case, is under-determined, since the quarks differ by localization, but not necessarily by the highest weights of their gauge representations.

(iv) In order to account for the hypercharges of the leptons and Higgses, and for the absence of chiral exotics, we set up necessary, but in general not sufficient, linear constraints:

$$
\begin{array}{rlrl}
\sum_{(\mathbf{3}, \mathbf{2}),(\overline{\mathbf{3}}, \mathbf{2})} Y & =1, & & \sum_{(\mathbf{3}, \mathbf{1}),(\overline{\mathbf{3}}, \mathbf{1})} Y=-2, \\
\sum_{(\mathbf{1}, \mathbf{2})} Y=-3, & & \sum_{(\mathbf{1}, \mathbf{1})} Y=6 .
\end{array}
$$

For readability, we use $Y$ in the above equations as a shorthand for $\Lambda_{k} \cdot Y$, where $\Lambda_{k}$ runs over the highest weights of the representations in the sum.

Note that the sum over e.g. the $(\mathbf{3}, \mathbf{1})$ and $(\overline{\mathbf{3}}, \mathbf{1})$ representations reduces to that over the right-handed quarks $\bar{u}$ and $\bar{d}$ alone, since we assume pairs of exotic particles to carry hypercharge assignments that are equal in magnitude but opposite in sign.

(v) Another constraint comes from the requirement that hypercharge be nonanomalous. If the model has an anomalous $\mathrm{U}(1)$ direction that we will denote by $\mathrm{U}_{1 A}$, we demand that it be orthogonal to $Y$ :

$$
\left(x_{1} U_{1}+x_{2} U_{2}+\ldots+x_{n} U_{n}\right) \cdot \mathrm{U}_{1 A}=0
$$

(vi) For solving the obtained system of linear diophantine equations, we developed $\mathrm{C}++$ code implementing the algorithms described in Ref. [18].

(vii) The solution to the system of linear diophantine equations is given by the sum of a particular solution and a linear combination of (not necessarily linearly independent, see Ref. [18]) homogeneous solutions, ${ }^{8}$ where the coefficients are all integers. In most cases, there are no more than 2 homogeneous solutions, so that we can simply iterate over the integer coefficients to obtain candidates for hypercharge. Each candidate will surely fulfill Eqs. (7)-(9), but may fail to give the correct hypercharge values on the leptons and Higgses; furthermore, the spectrum may not be vectorlike.

(viii) Some remarks are in order. First, note that we explicitly make use of the coefficients being integers, which is an assumption that is not fully justified. Second, we can of course not loop over all integer coefficients, but only over a finite subset. The lattice basis reduction [19] ensures that the integer coefficients are "small." Nevertheless, by restricting ourselves to a finite set, we may miss an interesting

\footnotetext{
${ }^{8}$ For efficiency, we minimized the length of the particular solution, and applied an LLL lattice basis reduction [19] on the homogeneous solutions using the NTL libraries [20].
}

solution. The general ansatz presented in Sec. III B will not suffer from these restrictions.

A detailed discussion of our results is presented in Sec, IV C. At this point, let us briefly remark that we applied the strategy outlined above to the 1767 threegeneration ${ }^{9}$ models. For 1114 models, we could establish the existence of a suitable hypercharge direction. Since a negative answer in the case of the remaining 653 models does not prove that hypercharge does not exist, we will investigate these models using the more sophisticated tools described in Sec. III B.

\section{B. The general case with nonlinear constraint equations}

We now turn our attention to the most general ansatz for constructing a suitable hypercharge direction for which we have a vectorlike spectrum.

(i) Again, we make an ansatz

$$
Y=x_{1} U_{1}+x_{2} U_{2}+\ldots+x_{n} U_{n}
$$

for the hypercharge direction in terms of the Abelian group factors and demand that the linear constraints Eqs. (7)-(9) be satisfied.

(ii) The linear equations by no means exhaust the constraints we may require to be fulfilled by a vectorlike spectrum. In particular, there are the cubic,

$$
\begin{aligned}
\sum_{(\mathbf{3}, \mathbf{2}),(\overline{\mathbf{3}}, \mathbf{2})} Y^{3} & =\frac{1}{9}, & \sum_{(\mathbf{3}, \mathbf{1}),(\overline{\mathbf{3}}, \mathbf{1})} Y^{3} & =-\frac{56}{9}, \\
\sum_{(\mathbf{1}, \mathbf{2})} Y^{3} & =-3, & \sum_{(\mathbf{1}, \mathbf{1})} Y^{3} & =24,
\end{aligned}
$$

and the quintic,

$$
\begin{array}{rlrl}
\sum_{(\mathbf{3}, \mathbf{2}),(\mathbf{3}, \mathbf{2})} Y^{5} & =\frac{1}{81}, \quad \sum_{(\mathbf{3}, \mathbf{1}),(\mathbf{3}, \mathbf{1})} Y^{5}=-\frac{992}{81}, \\
\sum_{(\mathbf{1}, \mathbf{2})} Y^{5} & =-3, \quad & \sum_{(\mathbf{1}, \mathbf{1})} Y^{5}=96,
\end{array}
$$

constraints. As before, the sum over e.g. all $(\mathbf{1}, \mathbf{1})$ representations reduces to that of the right-handed electrons that carry hypercharge +2 , since the righthanded neutrinos have zero hypercharge, and the exotic particles come in vectorlike pairs so that their contribution to the sum vanishes.

(iii) It is important to note that in this approach we consider the linear and the nonlinear equations on the same footing. In particular, we do not assume that the linear equations are diophantine. This fact adds to the calculational complexity of the problem, which scales with the number of variables.

\footnotetext{
${ }^{9}$ This number is slightly higher than the 1170 models listed in Ref. [1], since we lifted the constraint that the standard model gauge symmetry be a subset of SO(10). See Sec. IV C for more details.
} 
(iv) As in the case of the linear constraints, we scale $Y$ by $3 \mathrm{~N}$, so that the coefficients in the polynomial equations are guaranteed to be integers. This is more calculational convenience than a conceptional necessity, since the computational tools we are using and that are to be described below have a far better performance for integer arithmetics.

(v) The fact that these equations are highly nonlinear may at first seem discouraging. Luckily, there are efficient methods for solving systems of polynomial equations. The first step is the calculation of a Gröbner basis [21], from which we can already read off the dimensionality of the solution set, and, in particular, whether the system has a solution at all. To this end, we used the computer algebra system SINGULAR [22]. We present the details of the calculation in Appendix A.

(vi) If there are no solutions to the constraint equations, this rigorously proves the absence of a U(1) that may play the role of hypercharge. In the cases where solutions exist, we determine them numerically using Laguerre's algorithm as implemented by SINGULAR [22]. Equation (6) then gives the corresponding hypercharge direction, for which we can explicitly check whether our criteria are satisfied or not.

(vii) It turns out that in all cases, where the linear, cubic, and quintic equations were satisfied, the solution gives a vectorlike spectrum. Demanding only the linear and cubic equations, however, does not guarantee that the spectrum is vectorlike, as we have learned from experience in the course of our investigations.

In conclusion, let us remark that solving the nonlinear constraint equations adds 106 models to the list of 1114 vectorlike spectra of three-generation standard models already constructed in Sec. III A, so that the total number increases to 1220 . Also, this approach rigorously proves that for 547 out of 1767 models, there is no $\mathrm{U}(1)$ direction that can play the role of hypercharge in a way that is compatible with low-energy physics. In Sec. IV, we discuss these results in greater detail.

\section{PHENOMENOLOGY OF THE MODELS}

\section{A. The mini-landscape revisited}

We consider a class of orbifold models with promising phenomenology [1]. In order to keep the discussion as clear as possible, we focus on one particular shift, namely $V^{\mathrm{SO}(10), 1}$, which incidentally gives the largest number of models.

The results of the mini-landscape search [1] relevant to our present discussion can be summarized as follows. The shift $V^{\mathrm{SO}(10), 1}$ breaks

$$
\mathrm{E}_{8} \times \mathrm{E}_{8}^{\prime} \rightarrow \mathrm{SO}(10) \times \mathrm{SU}(2)^{2} \times \mathrm{U}(1) \times \mathrm{SO}(14)^{\prime} \times \mathrm{U}(1)^{\prime} .
$$

If we allow for up to 2 Wilson lines, there are 21841 inequivalent models. What we mean by two models being inequivalent is explained in Sec. IV B.

The gauge symmetries of 7149 models contain $\mathrm{SU}(3) \times$ $\mathrm{SU}(2)$ as a subgroup in at least one $\mathrm{E}_{8}$, and thereof, 1767 models have a three-generation spectrum. Since we do not know hypercharge yet, three-generation model in this context means that the spectrum, considering only color and weak isospin, has the right number of representations that can be identified with the particle content of the standard model. For a specific example of such a model, see e.g. Table II in Sec. III. We will refer to it as a 3-2 standard model.

In the mini-landscape search [1], our rationale had been to construct a default candidate for hypercharge, namely, that of Georgi-Glashow $\mathrm{SU}(5) \subset \mathrm{SO}(10)$. To that end, we considered 3563 models whose gauge symmetries contained $\mathrm{SU}(3) \times \mathrm{SU}(2)$ as a subgroup of $\mathrm{SO}(10)$. Of this set, 1170 models had a three-generation spectrum w.r.t. the $\mathrm{SU}(3) \times \mathrm{SU}(2) \subset \mathrm{SO}(10)$ gauge symmetry. Taking hypercharge to be that of the Georgi-Glashow model, 127 models had a vectorlike spectrum (see Fig. 3). Here and in the following, we will call a three-generation model a 3-2-1 vectorlike standard model, if hypercharge has the correct values (cf. Table I) on the quarks, leptons, and Higgses, and if all the exotic states come in pairs such that the standard model quantum numbers including hypercharge in each pair are equal in magnitude but opposite in sign.

\section{B. The general search for hypercharge in the "Fertile Patch"}

The starting point of our search is the set of 21841 inequivalent models. We call 2 models equivalent, if their spectra coincide. In this context, there are a couple of nontrivial issues that must be addressed. (i) The unbroken gauge group is typically the product of several simple factors. For each such factor, we have the choice whether to read its Dynkin diagram from left to right or from right to left, which corresponds to swapping all the representations with the complex conjugate ones. Thus, 2 spectra that differ only by complex conjugation w.r.t. one or more gauge group factors are to be identified. (ii) Because of its highly symmetric Dynkin diagram, the algebra $\mathrm{SO}(8)$ deserves special attention. Although the representations $\mathbf{8}_{v}, \mathbf{8}_{s}, \mathbf{8}_{c}$ are not equivalent, their tensor products are unaffected by a cyclic permutation, so we identify models whose spectra coincide after this permutation. (iii) In most cases, the same gauge group factor appears multiple times in the gauge symmetry. We identify those models whose spectra coincide when permuting the identical factors in the same $\mathrm{E}_{8}$. (iv) Two models whose spectra are identical may differ in the localization of the particles. At present, 
we do not distinguish between those models. (v) When checking the equivalence of models, we consider only the non-Abelian charges. To what extent two models that coincide in all the non-Abelian quantum numbers may differ w.r.t. $U(1)$ charges is subject to further research.

In the present case we want to be as general as possible, so, in contrast to the mini-landscape search, we do not require that the standard model gauge group be contained in $\mathrm{SO}(10)$. There are 7149 models whose unbroken gauge group contains $\mathrm{SU}(3) \times \mathrm{SU}(2)$ as a subset of the first or second $\mathrm{E}_{8}$, and 1767 of them are 3-2 standard models, see Fig. 4.

For each of the 1767 models, we first identify all representations of the form $(\mathbf{3}, \mathbf{2})$ and $(\overline{\mathbf{3}}, \mathbf{1})$. Generically, the number of these representations is greater than 3 and 6 , respectively, so we must make a choice as to what we call left-handed quark doublets and right-handed up- and down-type antiquark singlets. For each such choice, we generate the linear, cubic, and quintic constraint equations along the lines of Sec. III. To get a flavor of the details of the calculation, the reader is referred to the example in Appendix A. Let us here briefly remark that we are making the most general ansatz in that we treat the linear, cubic, and quintic constraints on the same footing and that we do not assume that the equations are diophantine.

The following cases need to be distinguished. (i) There are only finitely many solutions. We pick any of the solutions and calculate $Y$, see Eq. (10). (ii) The solutions are given by continuous parameters, and the relations intertwining these parameters are linear. We specialize to a numerical solution by setting all independent parameters equal to zero. (iii) Again, the solutions are given by continuous parameters, but this time, the relations intertwining the parameters are nonlinear. Since we cannot easily solve for the independent parameters, we numerically find one special solution and calculate the corresponding $Y$. Hypercharge directions which lead to irrational charges for the exotics are discarded.

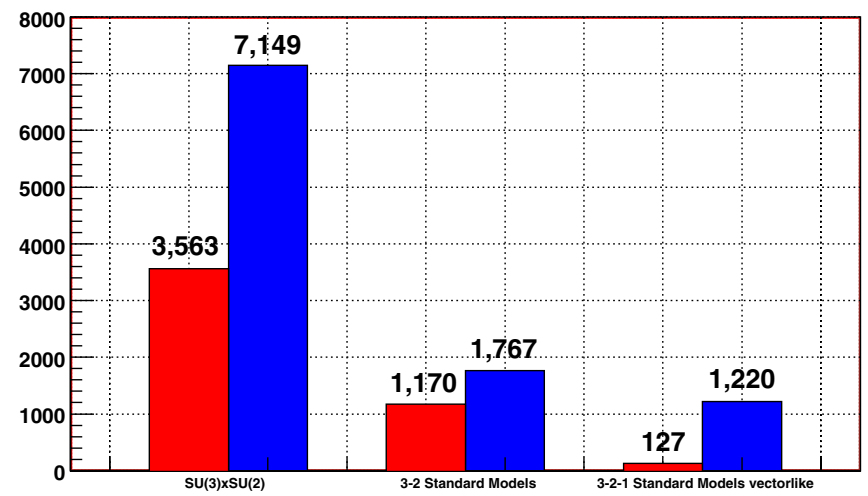

FIG. 4 (color online). Comparing the results of Ref. [1] (red) to our findings (blue). Dropping the assumption of hypercharge coming from an $\mathrm{SO}(10)$ leads to $10 \times$ more models.
Since our goal is to establish the existence of a suitable hypercharge direction, we stop at the first solution we find, i.e. as soon as the dimension of the ideal that describes the set of solutions to the system of polynomial equations is greater than or equal to zero.

Our findings are most unexpected. It turns out that 1220 models allow for a hypercharge direction for which the spectrum is vectorlike and contains three generations of quarks and leptons, i.e. we find 10 times more models than in the mini-landscape case, see Fig. 4. We were unsuccessful in our search only for 547 models. In Sec. IV C, we will analyze the 1220 vectorlike 3-2-1 standard models in greater detail.

\section{Analyzing the vectorlike models}

An important question is in what respect these 1220 vectorlike three-generation standard models differ from the 127 ones constructed previously, see Fig. 4. We will take a closer look at some key properties of the 127 models that are deemed desirable features for their phenomenology and see to what extent they are realized in the larger set.

\section{1126 Models: Standard model gauge group in SO(10)}

Remember that in Sec. IV we had dropped the assumption that the standard model gauge group be contained in $\mathrm{SO}(10)$. If we now reinstate this constraint, the number of vectorlike models decreases slightly from 1220 to 1126 (see Fig. 5). Based on the number of three-generation models in Fig. 4, we could have expected to see a more pronounced effect, i.e. a more dramatic drop. This suggests that the $\mathrm{SO}(10)$ structure enhances the chances of a model to have three generations.

A word of caution is in order. Because of the structure of the gauge symmetry breaking [see Eq. (13)], the SU(3) factor of the standard model must necessarily be in $\mathrm{SO}(10)$. As to the $\mathrm{SU}(2)$ factor, it may lie outside of $\mathrm{SO}(10)$, but can in many cases be related to one which lies in $\mathrm{SO}(10)$ by an

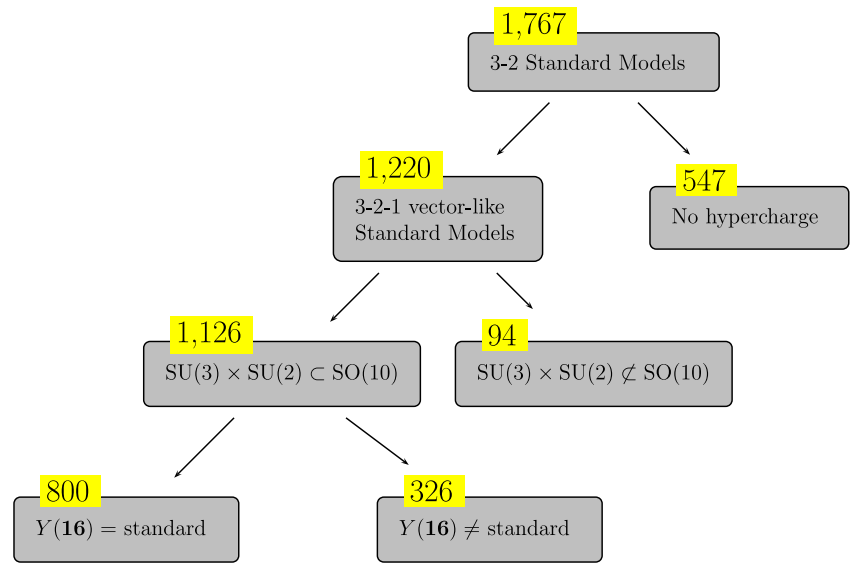

FIG. 5 (color online). Overview over the fraction of models passing our criteria. 
outer automorphism, see Appendix D for a specific example. Thus, we may be tracing a feature that is mathematical in nature and bears no physical relevance.

The same example in Appendix D illustrates a point which we had not anticipated. Two distinct sets of shifts and Wilson lines may lead to the same gauge group and spectrum, but to different symmetry breaking patterns. The consequences of this observation are rather bizarre. In the case of the first model in Appendix D, the symmetry breaking pattern allows us to complete the standard model gauge group to an intermediate $\mathrm{SU}(5)$ and consequently to derive Georgi-Glashow hypercharge, for which the spectrum is vectorlike and has three generations of quarks and leptons. As such, it qualifies for our list of phenomenologically promising models. In contrast, in the second model, there is no such intermediate $\mathrm{SU}(5)$ that we can use to construct a default hypercharge direction. ${ }^{10}$

In the early stages of our analysis, we had found only 1094 models, whose standard model gauge group was a subset of $\mathrm{SO}(10)$. After becoming aware of the subtleties described in the previous paragraph, we took a closer look at the 126 models which, loosely speaking, were not a subset of $\mathrm{SO}(10)$. In 32 cases, we could find a "twin model" such that the spectra coincide and the gauge group is indeed a subset of $\mathrm{SO}(10)$, thus giving us 1126 models in total, see Fig. 5.

\section{127 Models: Hypercharge direction purely due to SO(10)}

Next we would like to know how the 127 vectorlike three-generation models of the mini-landscape [1] fit into this new picture. Clearly, we expect them to be a subset of the 1126 models, and this is easily verified. By construction, these 127 models satisfy

$$
Y_{\text {general }}-Y_{\mathrm{GG}} \equiv 0,
$$

and thus give the standard hypercharges on the $\mathbf{1 6}$ of $\mathrm{SO}(10)$. We will now generalize this desirable feature.

\section{800 Models: Not purely SO(10), but standard charges on 16-plet}

After these preliminary considerations, we ask the question that is at the heart of the matter: Of the 1126 models, in those cases where $Y_{\text {general }}$ is different from $Y_{\mathrm{GG}}$, how does this difference affect the spectrum?

Despite this difference not being zero, the presence of $\mathrm{SO}(10)$ is too conspicuous to be ignored. We expect that the 16-plets in the first twisted sector will still give rise to 2 of the 3 families, and our best guess is that $Y_{\text {general }}$ is such that it coincides with $Y_{\mathrm{GG}}$ on these representations.

\footnotetext{
${ }^{10}$ In the case of the mini-landscape study, this was not a problem, since we first constructed Georgi-Glashow hypercharge and then removed equivalent models. As a result, we ran over all models.
}

Indeed, calculating $Y_{\mathrm{GG}}$ for the 1126 models where we have an intermediate SU(5) and comparing it to $Y_{\text {general }}$, we find that in 800 cases, it coincides with $Y_{\mathrm{GG}}$ when evaluated on the 16-plet:

$$
Y_{\text {general }}-Y_{\mathrm{GG}} \neq \equiv 0, \quad\left(Y_{\text {general }}-Y_{\mathrm{GG}}\right)(\mathbf{1 6}) \equiv 0
$$

In Appendix B, we present an explicit example. The two hypercharge directions $Y_{\mathrm{GG}}$ and $Y_{\text {general }}$, given in Eq. (B4) and (B5), respectively, differ only in entries in the second $\mathrm{E}_{8}$. As such, they give the same hypercharge on particles coming from the $\mathbf{1 6}$ of $\mathrm{SO}(10)$ that lies completely in the first $\mathrm{E}_{8}$, but do change the hypercharge on the other states. Comparing the two spectra for the same shift and Wilson lines, but different choices for hypercharge, namely, either $Y_{\mathrm{GG}}$ or $Y_{\text {general }}$, we find that in the former case, the model not only fails to be vectorlike, but does not even contain three generations, see Appendix B for the details. On the other hand, taking hypercharge to be $Y_{\text {general }}$, we get a three-generation model that is vectorlike.

We expect that these 800 models will inherit most of the properties of the 127 ones derived in Ref. [1]. The question of gauge coupling unification is the exception from the rule: The extra components of $Y_{\text {general }}$ lying outside $\mathrm{SO}(10)$ will have an impact on the value of the GUT scale. We will address this question in Sec. IV D.

\section{326 Models: Families do not form complete multiplets of $S O(10)$}

In the remaining 326 vectorlike three-generation models, it is no longer true that two families arise from 16's of $\mathrm{SO}(10)$ in the first twisted sector, i.e. these models are structurally different from the 127 ones we considered so far as paradigms.

We looked at one example in detail. In summary, calculating the values of $Y_{\text {general }}$ on the 16-plet at the origin in the first twisted sector, we find that the states corresponding to $\bar{u}$ and $\bar{e}$ are missing. Perusing the four-dimensional spectrum, it turns out that the representation playing the role of the $\bar{u}$ lives at a fixed point in the first twisted sector with no $\mathrm{SO}(10)$ symmetry, whereas $\bar{e}$ lives at the origin, but arises from a doublet representation outside of $\mathrm{SO}(10)$.

\section{Gauge coupling unification}

The value of $\sin ^{2} \theta_{w}$ deserves some special attention, because for one thing, it sets the GUT scale in the theory, and, as a consequence, it bears direct relevance to observable parameters at the electroweak scale.

At the GUT scale, the coupling constants $g$ and $g^{\prime}$ are equal, if the generators of $\mathrm{SU}(2)_{L}$ and $\mathrm{U}(1)_{Y}$ are normalized in the same way. In our conventions, where

$$
Q=T_{3 L}+\frac{Y}{2},
$$

there is a constant of proportionality, $g=C g^{\prime}$, which leads 
to

$$
\sin ^{2} \theta_{w}=\frac{g^{\prime 2}}{g^{2}+g^{\prime 2}}=\frac{1}{1+C^{2}} .
$$

The constant is given by the relative normalization of the generators,

$$
\left\|\frac{Y}{2}\right\|=C\left\|T_{3 L}\right\| .
$$

Up to a factor, the norm is given by the Killing form, see e.g. $§ 13.1 .2$. of Ref. [23]. Since the vector $Y$ represents the sum over Cartan generators with the respective coefficients, the norm of the generator corresponding to $Y$ reduces to its vector norm. Evaluating the Killing form on the fundamental representation for $\mathrm{SU}(2)_{L}$ canonically used in particle physics gives $\left\|T_{3 L}\right\|^{2}=1 / 2$.

We calculated $\sin ^{2} \theta_{w}$ for the 1220 models (see Fig. 5) for which a hypercharge direction exists. The results are presented in Fig. 6. For a judicious assessment of the data, the following remarks should be given some consideration.

For a given model, there is typically more than one hypercharge direction. In our general search in Sec. IV C, we stopped at the first solution that gave a vectorlike 3-2-1 standard model. In most of the models, $\sin ^{2} \theta_{w}$ is not only not equal to the standard GUT value of $3 / 8$, but it is generically too small.

Aiming at a higher value of $\sin ^{2} \theta_{w}$, our first ansatz has been to minimize the norm of $Y$. If there are only finitely many solutions to the constraint equations, we construct each hypercharge direction and pick the one with smallest norm. In many cases, however, the set of solutions is given by one or more parameters. In those cases where the parameters are given by linear relations, we use a simplex algorithm [24] to find $Y$ with minimal norm. The algorithm requires a "best guess" as input and in some cases failed to find the minimal solution. There were also a couple of cases where the solutions were parameterized by nonlinear equations, adding to the complexity of a numerical minimization.

For this and other reasons, we decided to specifically search for those models which have a hypercharge direc-

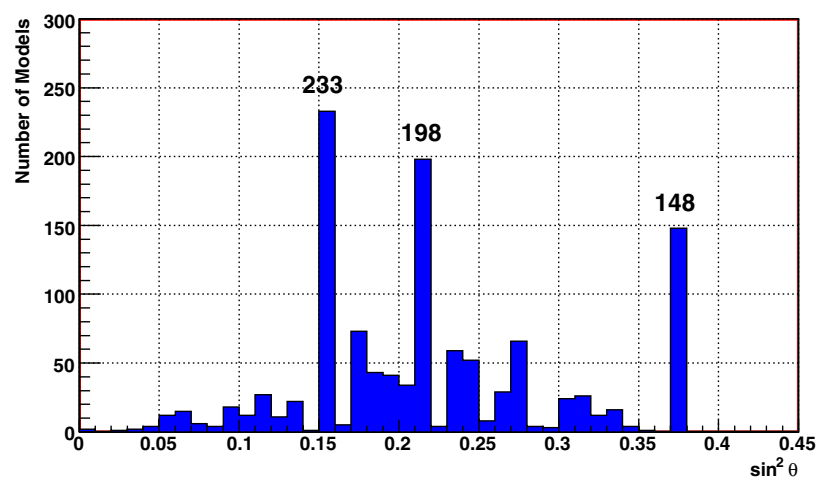

FIG. 6 (color online). The value of $\sin ^{2} \theta_{w}$ at the GUT scale for the 1220 vectorlike models. tion such that $\sin ^{2} \theta_{w}=3 / 8$. Bearing the results of Sec. III in mind, it is straightforward to implement this constraint [see Eq. (6) for $Y$ in terms of the independent $\mathrm{U}(1)$ directions]:

$$
\sin ^{2} \theta_{w}=\frac{3}{8} \leftrightarrow\|Y\|^{2}=\frac{10}{3} \leftrightarrow \sum_{i, j} x_{i} x_{j} U_{i} \cdot U_{j}=\frac{10}{3}
$$

We simply add Eq. (19) to the set of constraint equations in Secs. III A and III B and search for solutions. Naturally, we can restrict ourselves to the 1220 vectorlike standard models for which we know that hypercharge exists.

The results are presented in Fig. 6 . The values for $\sin ^{2} \theta_{w}$ in the histogram are "biased" in the sense that first, we specifically looked for the correct GUT scale relation, and second, we picked the smallest value whenever the problem was amenable to numeric minimization. Had we listed the values without these priors, we would have more models with lower values of $\sin ^{2} \theta_{w}$.

For 148 models, we find $\sin ^{2} \theta_{w}=3 / 8$. This number is to be compared to the 127 models of the mini-landscape (see Fig. 4), where hypercharge is constructed from $\mathrm{SO}(10)$ and the constraint on $\sin ^{2} \theta_{w}$ is thus satisfied by construction. In the following we take a closer look at the 21 extra models to assess in what respect, if at all, they differ from the 127 ones.

In 12 cases, hypercharge has components both in the first and in the second $E_{8}$. An explicit check shows that the hypercharge assignments for the particles coming from the 16 of $\mathrm{SO}(10)$ are nonstandard in all cases. This was to be expected, since the norm of hypercharge is the same as in the Georgi-Glashow case, but there exist components outside of $\mathrm{SO}(10)$. In 5 cases, $\mathrm{SU}(3) \times \mathrm{SU}(2) \subset \mathrm{SO}(10)$. To see how this fits into our present classification of the cases, see Fig. 5.

In 6 cases, hypercharge lives in the second $E_{8}$ and as such, the standard model is not a subset of the $\mathrm{SO}(10)$ that is left over after the first symmetry breaking due to the shift alone. There is a fair chance that hypercharge may be in some $\mathrm{SO}(10)^{\prime} \subset \mathrm{SO}(14)^{\prime} \subset \mathrm{E}_{8}^{\prime}$, see Eq. (13), so we are following this lead. Indeed, in 5 cases the symmetry breaking pattern is

$$
\mathrm{E}_{8}^{\prime} \stackrel{V}{\rightarrow} \mathrm{SO}(14)^{\prime} \stackrel{W_{3}}{\rightarrow} \mathrm{SU}(5)^{\prime} \times \mathrm{SU}(2)^{\prime} \times \mathrm{SU}(2)^{\prime} \stackrel{W_{2}}{\rightarrow} \mathrm{SM} \times \text { hidden, }
$$

and, moreover, hypercharge lies completely in $\mathrm{SU}(5)^{\prime}$, so we are back to the Georgi-Glashow case. ${ }^{11}$ In the one remaining case, the standard model is a subgroup of $\mathrm{SU}(4)^{\prime} \times \mathrm{SU}(4)^{\prime}$.

Most interestingly, in 3 cases hypercharge is all in the first $\mathrm{E}_{8}$, but does not arise from Georgi-Glashow. The nonAbelian gauge group factors of the standard model are not a subset of $\mathrm{SO}(10)$, and in 2 cases the $\mathrm{SU}(3)$ and $\mathrm{SU}(2)$

\footnotetext{
${ }^{11}$ Note, these differ from the 127 , since the Georgi-Glashow there was required to lie in the first $\mathrm{E}_{8}$.
} 
factors are not only in different $\mathrm{E}_{8}$ 's, but the constructed hypercharge gives the standard values on the $\mathbf{1 6}$ of $\mathrm{SO}(10)$. This observation is interesting enough to present the full details in Appendix E.

\section{CONCLUSIONS}

It has long been debated whether string theory is a theory of everything or anything, and the present work tries to shed some light on this important question. Admittedly, we have worked out the details for a very special class of string theory models, namely, orbifold compactifications of the heterotic string. Despite all the qualifications which will necessarily apply to our results, the big advantage of our ansatz is that we have a complete picture in the sense that we calculated all models in a welldefined framework and based our conclusions on finite numbers, thereby avoiding any discussion of a measure in the space of vacua.

One result of our studies is that for $69 \%$ of the 3-2 Standard models, there exists a hypercharge direction such that the spectrum becomes a 3-2-1 standard model and all exotics are vectorlike. This feature had hitherto been attributed to hypercharge arising from GeorgiGlashow SU(5) and has now been found to be a more general result. About $66 \%$ (or 800 of 1220) of the 3-2-1 standard models have the same characteristic features as those found in the mini-landscape search [1] with the exception of the Weinberg angle at the GUT scale.

The basic question we tried to answer in this publication can be summarized as follows: Given the standard model quantum numbers and the absence of chiral exotics at low energies, do orbifold compactifications predict or at least prefer values of $\sin ^{2} \theta_{w}$ compatible with grand unification? In other words, in how many cases is the standard GUT value realized and to what extent is it correlated to the presence of an $\mathrm{SO}(10)$ symmetry? Our results clearly indicate that the most preferred value of $\sin ^{2} \theta_{w}$ is in gross disagreement with grand unification and leads to a GUT scale which is too low. Nevertheless, a sizable fraction of the models attain the standard GUT value which lets us hope that there is some interesting physics yet to be explored.

Realizing that it might have been too naive to expect string theory to predict the Weinberg angle, we can add $\sin ^{2} \theta_{w}=3 / 8$ to our list of priors and ask, given the standard model quantum numbers, the absence of chiral exotics at low energies and the Weinberg angle, do we necessarily have an $\mathrm{SO}(10)$ as a local symmetry? In this case, we find a strong correlation between $\sin ^{2} \theta_{w}=3 / 8$ and an $\mathrm{SO}(10)$ being realized at an intermediate level in higher dimensions. In fact, of the models with the standard model spectrum, vectorlike hypercharge and $\sin ^{2} \theta=3 / 8$, $89 \%$ have $\mathrm{U}(1)_{Y} \subset \mathrm{SU}(5) \subset \mathrm{SO}(10)$ in the first or second $\mathrm{E}_{8}$. However, it came somewhat as a surprise that there exist 16 models (11\%) which do not quite fit into the usual
GUT picture and are nevertheless consistent with unification. Moreover, there are 21 models in excess of the minilandscape results [1] which are a priori phenomenologically viable and should not be left out of consideration in the quest for realistic models.

Let us remark that the methods outlined in the present publication have a broader range of application. These include the search for (gauged) discrete family symmetries, for Peccei-Quinn-type symmetries for the $\mu$-problem, and for a suitable B-L symmetry, a discrete subgroup of which can play the role of $R$-parity. The latter case has already been worked out and the results will be presented in a follow-up publication to the mini-landscape project [25].

The question of hypercharge normalization in the context of string theory has been addressed before, see e.g. Ref. [26] and references therein. We are aware of the wellknown discrepancy between unification at a string scale of order $10^{17} \mathrm{GeV}$ and gauge coupling unification which occurs at $10^{16} \mathrm{GeV}$. There are, however, several reasons to believe that this discrepancy will be resolved by threshold corrections from Kaluza-Klein modes between a compactification scale and string scale, see e.g. Refs. [27-30] for orbifold GUTs and Refs. [2,4,31,32] for string threshold corrections with asymmetrically large moduli. In either case the resolution comes from having grand unification in the extra dimensions and the standard model in 4 dimensions.

Finally, a general search on a specific class of $\mathbb{Z}_{3}$ orbifolds has been performed in Ref. [33]. In this analysis, the author looks for standard model gauge structure with three families of quarks and leptons having the correct quantum numbers. In most cases, this requires a generalized hypercharge not in SU(5) unlike what we find. However, it is difficult to compare our analysis to his, because he does not study those cases with $\sin ^{2} \theta_{w}=3 / 8$ in any detail, and in the general search, he does not distinguish between models with vectorlike vs chiral exotics. In contrast, we first find the vectorlike models with suitable hypercharge and then analyze $\sin ^{2} \theta_{w}$.

\section{ACKNOWLEDGMENTS}

We would like to thank James Gray and Nathan Salwen for useful discussions, and Angela Doerschlag for carefully reading the draft. We are very much indebted to the Ohio Supercomputing Center for using their resources. This work was supported in part by the Department of Energy under Grant No. DOE/ER/01545-873.

\section{APPENDIX A: CONSTRUCTING HYPERCHARGE FOR A SPECIFIC MODEL}

We focus on one of the 21841 models for shift $V^{\mathrm{SO}(10), 1}$ presented in Ref. [1], which we will henceforth denote by $V_{6}$. Additionally, we have two Wilson lines, namely $W_{3}$ of 
order 3 and $W_{2}$ of order 2:

$$
\begin{aligned}
V_{6} & =\left(\begin{array}{llllllllllllllll}
\frac{2}{3} & -\frac{2}{3} & \frac{7}{6} & \frac{1}{6} & 0 & 0 & 0 & 0 & \frac{1}{3} & 0 & 0 & 0 & 0 & 0 & 1 & -1
\end{array}\right) \\
W_{3} & =\left(\begin{array}{llllllllllllllll}
-\frac{1}{6} & \frac{1}{6} & -\frac{1}{6} & -\frac{5}{6} & \frac{1}{6} & \frac{1}{6} & \frac{1}{6} & -\frac{1}{6} & \frac{2}{3} & 0 & -\frac{1}{3} & -\frac{1}{3} & -\frac{1}{3} & -\frac{1}{3} & -\frac{4}{3} & -\frac{2}{3}
\end{array}\right) \\
W_{2} & =\left(\begin{array}{llllllllllllllll}
-\frac{1}{2} & -1 & 1 & 0 & \frac{1}{2} & -1 & 0 & 0 & 0 & -\frac{1}{2} & 0 & 0 & 0 & 0 & 0 & -\frac{1}{2}
\end{array}\right)
\end{aligned}
$$

The shift and Wilson lines break

$$
\mathrm{E}_{8} \times \mathrm{E}_{8}^{\prime} \rightarrow \mathrm{SU}(3) \times \mathrm{SU}(2) \times \mathrm{U}(1)^{5} \times \mathrm{SU}(5)^{\prime} \times \mathrm{U}(1)^{\prime 4},
$$

\begin{tabular}{|c|c|c|c|}
\hline $3 \times(3,2,1)$ & $12 \times(\overline{\mathbf{3}}, \mathbf{1}, \mathbf{1})$ & $29 \times(\mathbf{1}, \mathbf{2}, \mathbf{1})$ & $8 \times(\mathbf{1}, \mathbf{1}, \mathbf{5})$ \\
\hline & $6 \times(\mathbf{3}, \mathbf{1}, \mathbf{1})$ & $136 \times(\mathbf{1}, \mathbf{1}, \mathbf{1})$ & $8 \times(\mathbf{1}, \mathbf{1}, \overline{\mathbf{5}})$ \\
\hline
\end{tabular}

and the spectrum is given by (omitting the $\mathrm{U}(1)$ charges):

The simple roots of the unbroken gauge group are:

$$
\begin{aligned}
& \alpha_{1}=\left(\begin{array}{llllllllllllllll}
0 & 0 & 0 & 0 & 0 & 0 & 0 & 0 & 0 & 0 & 0 & 0 & 0 & 1 & -1 & 0
\end{array}\right) \\
& \alpha_{2}=\left(\begin{array}{llllllllllllllll}
0 & 0 & 0 & 0 & 0 & 0 & 0 & 0 & 0 & 0 & 0 & 0 & 1 & -1 & 0 & 0
\end{array}\right) \\
& \alpha_{3}=\left(\begin{array}{llllllllllllllll}
0 & 0 & 0 & 0 & 0 & 0 & 0 & 0 & 0 & 0 & 0 & 1 & -1 & 0 & 0 & 0
\end{array}\right) \\
& \alpha_{4}=\left(\begin{array}{llllllllllllllll}
0 & 0 & 0 & 0 & 0 & 0 & 0 & 0 & 0 & 0 & 1 & -1 & 0 & 0 & 0 & 0
\end{array}\right) \\
& \alpha_{5}=\left(\begin{array}{llllllllllllllll}
0 & 0 & 0 & 0 & 0 & 0 & 1 & 1 & 0 & 0 & 0 & 0 & 0 & 0 & 0 & 0
\end{array}\right) \\
& \alpha_{6}=\left(\begin{array}{llllllllllllllll}
0 & 0 & 0 & 0 & 0 & 1 & -1 & 0 & 0 & 0 & 0 & 0 & 0 & 0 & 0 & 0
\end{array}\right) \\
& \alpha_{7}=\left(\begin{array}{llllllllllllllll}
\frac{1}{2} & -\frac{1}{2} & -\frac{1}{2} & -\frac{1}{2} & -\frac{1}{2} & -\frac{1}{2} & -\frac{1}{2} & -\frac{1}{2} & 0 & 0 & 0 & 0 & 0 & 0 & 0 & 0
\end{array}\right)
\end{aligned}
$$

The $\mathrm{U}(1)$ directions are given by:

$$
\begin{aligned}
& U_{1}=\left(\begin{array}{llllllllllllllll}
0 & 0 & 0 & 1 & -1 & 0 & 0 & 0 & 0 & 0 & 0 & 0 & 0 & 0 & 0 & 0
\end{array}\right) \\
& U_{2}=\left(\begin{array}{llllllllllllllll}
0 & 0 & 1 & -1 & 0 & 0 & 0 & 0 & 0 & 0 & 0 & 0 & 0 & 0 & 0 & 0
\end{array}\right) \\
& U_{3}=\left(\begin{array}{llllllllllllllll}
0 & 1 & -1 & 0 & 0 & 0 & 0 & 0 & 0 & 0 & 0 & 0 & 0 & 0 & 0 & 0
\end{array}\right) \\
& U_{4}=\left(\begin{array}{llllllllllllllll}
0 & 0 & 0 & 0 & 0 & 0 & 0 & 0 & \frac{1}{2} & -\frac{1}{2} & -\frac{1}{2} & -\frac{1}{2} & -\frac{1}{2} & -\frac{1}{2} & -\frac{1}{2} & \frac{1}{2}
\end{array}\right) \\
& U_{5}=\left(\begin{array}{llllllllllllllll}
0 & 0 & 0 & 0 & 0 & 0 & 0 & 0 & 0 & 0 & 0 & 0 & 0 & 0 & 0 & -2
\end{array}\right) \\
& U_{6}=\left(\begin{array}{llllllllllllllll}
0 & 0 & 0 & 0 & 0 & 0 & 0 & 0 & 0 & 1 & 0 & 0 & 0 & 0 & 0 & -1
\end{array}\right) \\
& U_{8}=\left(\begin{array}{llllllllllllllll}
\frac{1}{2} & -\frac{1}{2} & -\frac{1}{2} & -\frac{1}{2} & \frac{1}{2} & \frac{1}{2} & \frac{1}{2} & -\frac{1}{2} & 0 & 0 & 0 & 0 & 0 & 0 & 0 & 0
\end{array}\right) \\
& U_{9}=\left(\begin{array}{llllllllllllllll}
0 & 0 & 0 & 0 & 0 & 0 & 0 & 0 & 0 & -2 & 1 & 1 & 1 & 1 & 1 & -1
\end{array}\right)
\end{aligned}
$$

\section{Linear diophantine equations}

It is easy to check that the $\mathrm{U}(1)$ directions are all orthogonal to the simple roots, and furthermore belong to the $\mathrm{E}_{8} \times \mathrm{E}_{8}^{\prime}$ lattice, thus satisfying criterion (i) in Sec. III A. For one particular choice of left- and right-handed quarks (which, due to space limitations, we cannot specify further, unless we list all the details of the spectrum, such as highest weights, localization, etc.), we obtain the following particular

$$
\tilde{U}_{0}=\left(\begin{array}{lllllllllllllllll}
-\frac{3}{2} & \frac{3}{2} & \frac{3}{2} & \frac{3}{2} & \frac{3}{2} & -\frac{5}{2} & -\frac{5}{2} & \frac{5}{2} & 0 & -6 & -1 & -1 & -1 & -1 & -1 & 1
\end{array}\right)
$$


and homogeneous solutions

$$
\begin{aligned}
& \tilde{U}_{1}=\left(\begin{array}{llllllllllllllll}
0 & 1 & 0 & -1 & 0 & 0 & 0 & 0 & -\frac{1}{2} & \frac{1}{2} & \frac{1}{2} & \frac{1}{2} & \frac{1}{2} & \frac{1}{2} & \frac{1}{2} & \frac{7}{2}
\end{array}\right) \\
& \tilde{U}_{2}=\left(\begin{array}{llllllllllllllll}
0 & -1 & 0 & 1 & 0 & 0 & 0 & 0 & \frac{1}{2} & -\frac{1}{2} & \frac{1}{2} & \frac{1}{2} & \frac{1}{2} & \frac{1}{2} & \frac{1}{2} & \frac{3}{2}
\end{array}\right)
\end{aligned}
$$

to the system of 14 equations corresponding to Eqs. (7)-(9) in Sec. III A. Hypercharge is then given by

$$
Y=\frac{1}{3}\left(\tilde{U}_{0}+x_{1} \tilde{U}_{1}+x_{2} \tilde{U}_{2}\right)
$$

for some $x_{1}, x_{2} \in \mathbb{Z}$. We loop over all $-2 \leq x_{1}, x_{2} \leq 2$ and check whether $Y$ is such that it gives the correct values on the quarks, leptons and Higgses, and whether the exotics are vectorlike. The first solution we find is

$$
x_{1}=1, \quad x_{2}=1,
$$

which corresponds to

$$
Y=\left(\begin{array}{lllllllllllllllll}
-\frac{1}{2} & \frac{1}{2} & \frac{1}{2} & \frac{1}{2} & \frac{1}{2} & -\frac{5}{6} & -\frac{5}{6} & \frac{5}{6} & 0 & -2 & 0 & 0 & 0 & 0 & 0 & 2
\end{array}\right) .
$$

The spectrum, now including hypercharge, reads

\begin{tabular}{lllll}
\hline \hline $3 \times(\mathbf{3}, \mathbf{2})_{1 / 3}$ & $5 \times(\overline{\mathbf{3}}, \mathbf{1})_{-4 / 3}$ & $7 \times(\overline{\mathbf{3}}, \mathbf{1})_{2 / 3}$ & $16 \times(\mathbf{1}, \mathbf{2})_{-1}$ & $45 \times(\mathbf{1}, \mathbf{1})_{2}$ \\
\hline $2 \times(\mathbf{3}, \mathbf{1})_{4 / 3}$ & $4 \times(\mathbf{3}, \mathbf{1})_{-2 / 3}$ & $13 \times(\mathbf{1}, \mathbf{2})_{1}$ & $42 \times(\mathbf{1}, \mathbf{1})_{-2}$ \\
\hline \hline
\end{tabular}

where we have counted the representations of the hidden gauge group as multiplicities for the standard model particles.

Several remarks are in order. First, let us note that, had we not minimized the length of the particular solution and applied an LLL reduction [19] to the homogeneous solutions, we would have had to cover a considerably larger set of integers for $x_{1}$ and $x_{2}$. From experience we know that the loss of time in this case can be considerable.

Second, the presented model is such that the standard model gauge group is a subset of $\mathrm{SO}(10)$, but that none of the U(1) directions stemming from the GUT gives a threegeneration model with vectorlike exotics. Under this aspect, the construction of a general hypercharge direction may give useful phenomenological insights.

\section{Linear diophantine equations and the cubic constraints}

Despite the fact that we were able to construct a suitable hypercharge direction using only the linear constraints, this is not true in general. To demonstrate the use of the nonlinear constraints, we pursue the present example further. Starting from the general solution Eq. (A2) and substituting it into the cubic constraint Eq. (11), we obtain a system of 4 polynomial equations ${ }^{12}$ in 2 variables, which are too complicated even to attempt to write them down. However, calculating a Gröbner basis ${ }^{13}$ yields the elegantly short and

\footnotetext{
${ }^{12}$ To generate and manipulate polynomial equations, we have found GINAC [34] to be a very useful tool.

${ }^{13}$ Giving an introduction to Gröbner bases is beyond the scope of the present publication. For a general introduction, see Refs. [35,36]. We would like to point out Ref. [37] that made us aware of Gröbner bases and their applications in physics.
}

simple form

$$
x_{1}^{2}-2 x_{1} x_{2}+x_{2}^{2}=0,
$$

from which we see that the system has a one-dimensional set of solutions, e.g. $x_{1}=x_{2}=1$, as found before. One should not be misled to think that satisfying the linear and cubic constraints is sufficient for the spectrum to be threegeneration and vectorlike. In the course of our work, we encountered cases in which the constraints were fulfilled, but nevertheless the spectrum failed to be three-generation or vectorlike.

\section{Linear diophantine equations, the cubic and the quintic constraints}

Finally, requiring that the quintic constraints Eq. (12) be satisfied leaves us with only a finite number of solutions. Again, the constraints are too long to list, but after computing the Gröbner basis, we obtain

$$
\begin{gathered}
x_{1}^{2}-2 x_{1} x_{2}+x_{2}^{2}=0, \\
70 x_{1} x_{2}^{4}-45 x_{2}^{5}+8 x_{1} x_{2}^{3}-13 x_{2}^{4}-156 x_{1} x_{2}^{2}+70 x_{2}^{3} \\
+8 x_{1} x_{2}+30 x_{2}^{2}+70 x_{1}+7 x_{2}-49=0, \quad(\mathrm{~A} 7) \\
875 x_{2}^{6}-450 x_{2}^{5}+2880 x_{1} x_{2}^{3}-5835 x_{2}^{4}-1728 x_{1} x_{2}^{2}+400 x_{2}^{3} \\
-5184 x_{1} x_{2}+7461 x_{2}^{2}+4032 x_{1}-6594 x_{2}+539=0 .
\end{gathered}
$$

The only solutions to this system are

$$
x_{1}=x_{2}=1 \text { and } x_{1}=x_{2}=-\frac{7}{5} .
$$

The first solution is the one we already know, and calculating the hypercharge direction corresponding to 
the second solution, we obtain

$$
Y=\left(\begin{array}{llllllllllllllllllll}
-\frac{1}{2} & \frac{1}{2} & \frac{1}{2} & \frac{1}{2} & \frac{1}{2} & -\frac{5}{6} & -\frac{5}{6} & \frac{5}{6} & 0 & -2 & -\frac{4}{5} & -\frac{4}{5} & -\frac{4}{5} & -\frac{4}{5} & -\frac{4}{5} & -2
\end{array}\right)
$$

leading to the spectrum

\begin{tabular}{lllll}
\hline \hline $3 \times(\mathbf{3}, \mathbf{2})_{1 / 3}$ & $5 \times(\overline{\mathbf{3}}, \mathbf{1})_{-4 / 3}$ & $16 \times(\mathbf{1}, \mathbf{2})_{-1}$ & $30 \times(\mathbf{1}, \mathbf{1})_{2}$ & $15 \times(\mathbf{1}, \mathbf{1})_{6 / 5}$ \\
\hline & $2 \times(\mathbf{3}, \mathbf{1})_{4 / 3}$ & $13 \times(\mathbf{1}, \mathbf{2})_{1}$ & $27 \times(\mathbf{1}, \mathbf{1})_{-2}$ & $15 \times(\mathbf{1}, \mathbf{1})_{-6 / 5}$ \\
& $7 \times(\overline{\mathbf{3}}, \mathbf{1})_{2 / 3}$ & & $79 \times(\mathbf{1}, \mathbf{1})_{0}$ & $25 \times(\mathbf{1}, \mathbf{1})_{4 / 5}$ \\
& $4 \times(\mathbf{3}, \mathbf{1})_{-2 / 3}$ & & $25 \times(\mathbf{1}, \mathbf{1})_{-4 / 5}$ \\
\hline
\end{tabular}

which is a three-generation model with vectorlike exotics. Thus, we see that our assumption that the coefficients in Eq. (A2) be integers is not fully justified.

\section{The linear, lubic and quintic constraints on the same footing}

In light of these findings, we review our assumptions entering the foregoing calculation. Choosing the U(1) directions to be root vectors of $\mathrm{E}_{8} \times \mathrm{E}_{8}^{\prime}$ is safe, since we can always find $n$ such directions such that together with the roots of the unbroken gauge group they span the full 16dimensional lattice. For the linear constraints, we will obtain a system of equations with integer coefficients, but we must now drop the assumption that the solutions are given by integers, i.e. the system is not necessarily diophantine. For the cubic and quintic constraints, we had not assumed the solutions to be integers at all; therefore, there are no modifications.

In practice, this means that we cannot a priori reduce the system from 9 to 2 variables by solving the diophantine equations, but must rather consider the linear constraints on the same footing as the cubic and quintic ones. Starting with the general ansatz equation (6), we thus obtain a system of 12 polynomial equations in 9 variables. Pursuing the same calculations as previously outlined, one can show that there are exactly 2 solutions, corresponding to

$$
\begin{gathered}
x_{1}=x_{2}=x_{3}=x_{4}=x_{5}=x_{9}=0, \quad x_{6}=-6, \\
x_{7}=-1, \quad x_{8}=-2,
\end{gathered}
$$

and

$$
\begin{aligned}
x_{1}=x_{2} & =x_{3}=x_{4}=0, \quad x_{5}=\frac{48}{5}, \quad x_{6}=-\frac{54}{5}, \\
x_{7} & =-1, \quad x_{8}=-2, \quad x_{9}=-\frac{12}{5} .
\end{aligned}
$$

Using the nine $\mathrm{U}(1)$ directions listed at the beginning of this section, we recover exactly the 2 hypercharge directions which we have already constructed, thereby proving that these are the most general solutions.

\section{APPENDIX B: WHERE DID WE LOSE THE INTERESTING MODELS?}

Consider the model given at the beginning of Appendix A. We will now address the question to what extent the general hypercharge directions given in Eqs. (A4) and (A9) are connected to the presence of an underlying $\mathrm{SO}(10)$ symmetry. More specifically, we will explain why this model was not found by the analysis presented in Ref. [1], despite the fact that its standard model gauge group is contained in $\mathrm{SO}(10)$ and there exists a hypercharge direction such that its spectrum is vectorlike (see Appendix A).

For the following discussion, only the first $\mathrm{E}_{8}$ factor is relevant. The shift $V_{6}$ breaks

$$
\mathrm{E}_{8} \stackrel{V_{6}}{\rightarrow} \mathrm{SO}(10) \times \mathrm{SU}(2) \times \mathrm{SU}(2) \times \mathrm{U}(1)
$$

or in terms of Dynkin diagrams:

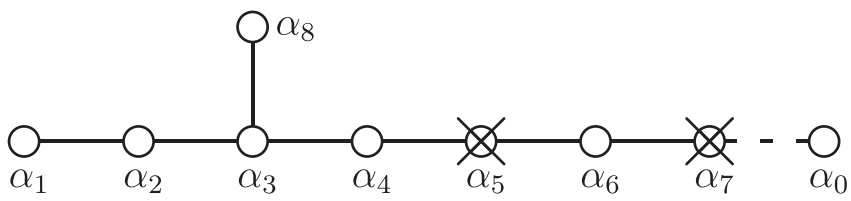

The Wilson lines $W_{3}$ and $W_{2}$ break $\mathrm{SO}(10)$ down to the standard model:

$$
\mathrm{SO}(10) \stackrel{W_{3}}{\rightarrow} \mathrm{SU}(4) \times \mathrm{SU}(2) \times \mathrm{U}(1) \stackrel{W_{2}}{\rightarrow} \mathrm{SU}(3) \times \mathrm{SU}(2) \times \mathrm{U}(1)^{2}
$$

Again, this is most conveniently visualized in terms of the associated Dynkin diagram:

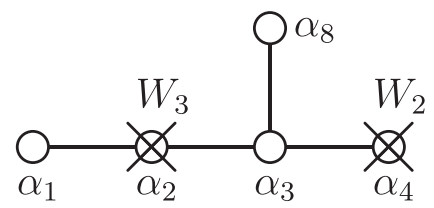

This clearly shows that the standard model gauge group is indeed a subset of $\mathrm{SO}(10)$. Nevertheless, perusing the list of 127 vectorlike models given in Ref. [1], we find that this model is missing. 
To see why we missed this model, we adopt the following strategy. First, we construct the standard $\mathrm{SO}(10)$ hypercharge as we did in Ref. [1]. Then, we compare this hypercharge to the general one, given in Appendix A, Eqs. (A4) and (A9).

Standard $\mathrm{SO}(10)$ hypercharge is a linear combination of the Cartan generators that lies in SU(5) and commutes with the $\mathrm{SU}(3) \times \mathrm{SU}(2)$ step operators. To find this hypercharge direction, we complete the standard model in all possible ways to an SU(5) symmetry. Up to a multiplicative factor, hypercharge will then be given by the dual of the root which completes the diagram. Having $\mathrm{SO}(10)$ in mind, we have a default choice:<smiles>O=COC(=O)OC=CO</smiles>

The hypercharge direction is then given by

$$
\begin{aligned}
\mathrm{U}(1)_{\mathrm{SO}(10)} & =\frac{5}{3} \alpha^{*}=\sum_{j} \frac{5}{3}\left(A_{\mathrm{SU}(5)}^{-1}\right)_{2 j} \alpha_{j} \\
& =\frac{1}{3}\left(3 \gamma+6 \alpha+4 \beta_{1}+2 \beta_{2}\right),
\end{aligned}
$$

or if we substitute for the $\mathrm{SU}(5)$ roots,

$$
Y_{\mathrm{GG}}=\left(\begin{array}{llllllllllllllll}
-\frac{1}{2} & \frac{1}{2} & \frac{1}{2} & \frac{1}{2} & \frac{1}{2} & -\frac{5}{6} & -\frac{5}{6} & \frac{5}{6} & 0 & 0 & 0 & 0 & 0 & 0 & 0 & 0
\end{array}\right) .
$$

Compare this result to the hypercharge direction found in Eq. (A4) in Appendix A as the result of a general search:

$$
Y_{\text {general }}=\left(\begin{array}{lllllllllllllllll}
-\frac{1}{2} & \frac{1}{2} & \frac{1}{2} & \frac{1}{2} & \frac{1}{2} & -\frac{5}{6} & -\frac{5}{6} & \frac{5}{6} & 0 & -2 & 0 & 0 & 0 & 0 & 0 & 2
\end{array}\right)
$$

\begin{tabular}{|c|c|c|c|c|c|}
\hline $3 \times(\mathbf{3}, \mathbf{2})_{1 / 3}$ & $4 \times(\overline{\mathbf{3}}, \mathbf{1})_{-4 / 3}$ & $3 \times(\overline{\mathbf{3}}, \mathbf{1})_{2 / 3}$ & $9 \times(\mathbf{1}, \mathbf{2})_{-1}$ & $2 \times(\mathbf{1}, \mathbf{1})_{2}$ & $103 \times(\mathbf{1}, \mathbf{1})_{0}$ \\
\hline & $2 \times(\mathbf{3}, \mathbf{1})_{-2 / 3}$ & $\begin{array}{l}4 \times(\mathbf{3}, \mathbf{1})_{0} \\
5 \times(\overline{\mathbf{3}}, \mathbf{1})_{0}\end{array}$ & $\begin{array}{l}5 \times(\mathbf{1}, \mathbf{2})_{1} \\
10 \times(\mathbf{1}, \mathbf{2})_{-1 / 3} \\
5 \times(\mathbf{1}, \mathbf{2})_{1 / 3}\end{array}$ & $\begin{array}{l}6 \times(\mathbf{1}, \mathbf{1})_{4 / 3} \\
6 \times(\mathbf{1}, \mathbf{1})_{-4 / 3} \\
37 \times(\mathbf{1}, \mathbf{1})_{2 / 3} \\
62 \times(\mathbf{1}, \mathbf{1})_{-2 / 3}\end{array}$ & \\
\hline
\end{tabular}

Remarkably, the two hypercharge directions differ only in entries in the second $E_{8}$. To assess what this means for the phenomenology of the model at hand, we recalculate the spectra, where in one case, we have chosen hypercharge to be $Y_{\mathrm{GG}}$,

and in the other case, we have chosen hypercharge to be $Y_{\text {general }}$ :

\begin{tabular}{llllll}
\hline \hline $3 \times(\mathbf{3}, \mathbf{2})_{-1 / 3}$ & $5 \times(\overline{\mathbf{3}}, \mathbf{1})_{-4 / 3}$ & $7 \times(\overline{\mathbf{3}}, \mathbf{1})_{2 / 3}$ & $16 \times(\mathbf{1}, \mathbf{2})_{-1}$ & $45 \times(\mathbf{1}, \mathbf{1})_{2}$ & $129 \times(\mathbf{1}, \mathbf{1})_{0}$ \\
\hline $2 \times(\mathbf{3}, \mathbf{1})_{-4 / 3}$ & $4 \times(\mathbf{3}, \mathbf{1})_{-2 / 3}$ & $13 \times(\mathbf{1}, \mathbf{2})_{1}$ & $42 \times(\mathbf{1}, \mathbf{1})_{-2}$ & \\
\hline \hline
\end{tabular}

In the case where hypercharge is purely $\mathrm{SO}(10)$, the spectrum fails to contain three generations of quarks and leptons and is not vectorlike either.

Looking at the top row of the first spectrum, it catches one's eye that the particle content corresponds to that of 2 complete 16-plets of $\mathrm{SO}(10)$, so our best guess is that 2 families still come from 16-plets localized at the first twisted sector and that the extra component of $Y_{\text {general }}$ does not affect these states at all. Indeed, this is easily verified by switching off the Wilson lines and evaluating $Y_{\text {general }}-Y_{\mathrm{GG}}$ on these 16-plets, which gives zero. The same difference is nonzero on more than half of the other states. In particular, it is nonzero on the right-handed electron of the third family, as a comparison of the two spectra shows. As for the remaining states of the third family, there exist standard model representations with the right quantum numbers on which this difference vanishes.

Flipped SU(5)

Consider the discussion preceding Eq. (B3). There is a second, not-so-obvious choice to complete the standard model Dynkin diagram to an SU(5) symmetry and subsequently to obtain a possible hypercharge direction:

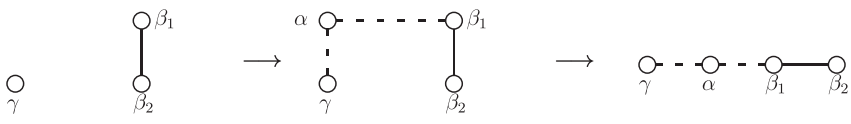

After a short calculation analogous to the one following Eq. (B3), we obtain:

$$
Y_{\text {flipped }}=\left(\begin{array}{lllllllllllllllll}
\frac{1}{2} & -\frac{1}{2} & -\frac{1}{2} & -\frac{1}{2} & \frac{3}{2} & \frac{1}{6} & \frac{1}{6} & -\frac{1}{6} & 0 & 0 & 0 & 0 & 0 & 0 & 0 & 0
\end{array}\right)
$$


This hypercharge direction corresponds to flipped $\mathrm{SU}(5)$, as can easily be shown by evaluating it on the $\mathbf{1 6}$ of $\mathrm{SO}(10)$. In comparison to $Y_{\mathrm{GG}}$, the roles of $\bar{u}, \bar{d}$ and of $\bar{e}$, $\bar{\nu}$ are interchanged.

\section{APPENDIX C: GRAND UNIFICATION FOR DUMMIES}

In this section, we give some useful background information on grand unification. The branching rules and $\mathrm{U}(1)$ charges that we present in Table III may either be looked up in some standard reference on group theory [14], or calculated by hand using explicit expressions for the simple roots in some standard basis, see e.g. Appendix 5A of Ref. [38].

\section{Georgi-Glashow SU(5)}

The matter content of the standard model fits in 3 irreducible representations,

$$
\begin{aligned}
& \mathbf{1 0} \rightarrow(\mathbf{3}, \mathbf{2})_{1 / 3}+(\overline{\mathbf{3}}, \mathbf{1})_{-4 / 3}+(\mathbf{1}, \mathbf{1})_{2}, \\
& \overline{\mathbf{5}} \rightarrow(\overline{\mathbf{3}}, \mathbf{1})_{2 / 3}+(\mathbf{1}, \mathbf{2})_{-1}, \quad \mathbf{1} \rightarrow(\mathbf{1}, \mathbf{1})_{0},
\end{aligned}
$$

where we have indicated the charges under $\mathrm{U}(1)_{Y}$ as subscripts. This hypercharge direction may be calculated by considering the Dynkin diagram associated with the symmetry breakdown:

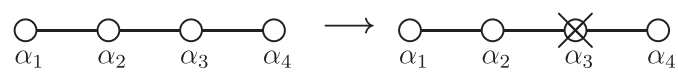

$\mathrm{U}(1)_{Y}$ is the dual of the root that is projected out. Keeping in mind that the dual roots are given in terms of the simple roots by the quadratic form matrix, which is the inverse of the Cartan matrix, we immediately obtain:

$$
\begin{aligned}
Y_{\mathrm{GG}} & =\frac{5}{3} \alpha_{3}^{*}=\frac{5}{3} \sum_{j=1}^{4}\left(A_{\mathrm{SU}(5)}^{-1}\right)_{3 j} \alpha_{j} \\
& =\frac{1}{3}\left(2 \alpha_{1}+4 \alpha_{2}+6 \alpha_{3}+3 \alpha_{4}\right)
\end{aligned}
$$

The $\mathrm{U}(1)_{Y}$ charges of the irreducible representations are calculated by taking the scalar product of the respective highest weight with $Y_{\mathrm{GG}}$. We summarize the results in the 4th column of Table III.

TABLE III. Branching rules and U(1) charges for the matter multiplets in the standard model.

\begin{tabular}{lcccccccc}
\hline \hline su(5) irrepSM irrep $U(1)_{X} U(1)_{Y} U(1)_{B-L} U(1)_{R} U(1)_{\mathrm{ff}}$ llippedGG \\
\hline $\mathbf{1 0}$ & $(\mathbf{3}, \mathbf{2})$ & -1 & $1 / 3$ & $1 / 3$ & 0 & $1 / 3$ & $Q$ & $Q$ \\
& $(\overline{\mathbf{3}}, \mathbf{1})$ & -1 & $-4 / 3$ & $-1 / 3$ & -1 & $2 / 3$ & $\bar{d}$ & $\bar{u}$ \\
& $(\mathbf{1}, \mathbf{1})$ & -1 & 2 & 1 & 1 & 0 & $\bar{\nu}$ & $\bar{e}$ \\
$\overline{\mathbf{5}}$ & $(\overline{\mathbf{3}}, \mathbf{1})$ & 3 & $2 / 3$ & $-1 / 3$ & 1 & $-4 / 3$ & $\bar{u}$ & $\bar{d}$ \\
& $(\mathbf{1}, \mathbf{2})$ & 3 & -1 & -1 & 0 & -1 & $L$ & $L$ \\
& $(\mathbf{1}, \mathbf{1})$ & -5 & 0 & 1 & -1 & 2 & $\bar{e}$ & $\bar{\nu}$ \\
\hline
\end{tabular}

\section{Pati-Salam}

The standard model particle content fits in one $\mathbf{1 6}$ of $\mathrm{SO}(10)$. Under $\mathrm{SO}(10) \rightarrow \mathrm{SU}(4)_{c} \times \mathrm{SU}(2)_{L} \times \mathrm{SU}(2)_{R}$, we have

$$
16 \rightarrow(4,2,1)+(\overline{4}, 1,2) .
$$

For more clarity, we break the Pati-Salam symmetry to that of the standard model in two steps. In the first step, we have

$$
\begin{aligned}
\mathrm{SU}(4)_{c} \times \mathrm{SU}(2)_{L} \times \mathrm{SU}(2)_{R} \rightarrow & \mathrm{SU}(3)_{c} \times \mathrm{U}(1)_{\mathrm{B}-\mathrm{L}} \\
& \times \mathrm{SU}(2)_{L} \times \mathrm{SU}(2)_{R}
\end{aligned}
$$

$$
\begin{aligned}
& (\mathbf{4}, \mathbf{2}, \mathbf{1}) \rightarrow(3,2,1)_{1 / 3}+(\mathbf{1}, \mathbf{2}, \mathbf{1})_{-1}, \\
& (\overline{\mathbf{4}}, \mathbf{1}, \mathbf{2}) \rightarrow(\overline{\mathbf{3}}, \mathbf{1}, \mathbf{2})_{-1 / 3}+(\mathbf{1}, \mathbf{1}, \mathbf{2})_{1},
\end{aligned}
$$

where the subscript denotes the $\mathrm{U}(1)_{\mathrm{B}-\mathrm{L}}$ charge. In the second step, we have

$$
\begin{aligned}
& \mathrm{SU}(3)_{c} \times \mathrm{U}(1)_{\mathrm{B}-\mathrm{L}} \times \mathrm{SU}(2)_{L} \times \mathrm{SU}(2)_{R} \\
& \rightarrow \mathrm{SU}(3)_{c} \times \mathrm{U}(1)_{\mathrm{B}-\mathrm{L}} \times \mathrm{SU}(2)_{L} \times \mathrm{U}(1)_{R}
\end{aligned}
$$

where the first subscript denotes the $\mathrm{U}(1)_{\mathrm{B}-\mathrm{L}}$ charge as above and the second one is the $\mathrm{U}(1)_{\mathrm{R}}$ charge. Hypercharge is then given as the linear combination

$$
\mathrm{U}(1)_{Y}=\mathrm{U}(1)_{\mathrm{B}-\mathrm{L}}+\mathrm{U}(1)_{R} \text {. }
$$

Again, this is most conveniently visualized in terms of Dynkin diagrams:

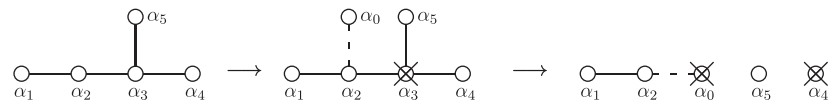

We can easily calculate the directions corresponding to the two Abelian factors:

$$
\begin{gathered}
Y_{\mathrm{B}-\mathrm{L}}=\frac{4}{3} \alpha_{0}^{*}=\frac{4}{3} \sum_{j=1,2,0}\left(A_{\mathrm{SU}(4)}^{-1}\right)_{0 j} \alpha_{j}=\frac{1}{3}\left(\alpha_{1}+2 \alpha_{2}+3 \alpha_{0}\right) \\
Y_{\mathrm{R}}=\alpha_{4}^{*}=\sum_{j=4}\left(A_{\mathrm{SU}(2)}^{-1}\right)_{4 j} \alpha_{j}=\frac{1}{2} \alpha_{4}
\end{gathered}
$$

Incidentally, we should remark that

$$
Y_{\mathrm{GG}}=Y_{\mathrm{B}-\mathrm{L}}+Y_{\mathrm{R}},
$$

as was to be expected.

\section{Flipped SU(5)}

Under $\mathrm{SO}(10) \rightarrow \mathrm{SU}(5) \times \mathrm{U}(1)_{X}$ we have

$$
\mathbf{1 6} \rightarrow \mathbf{1 0}_{-1}+\overline{\mathbf{5}}_{3}+\mathbf{1}_{-5} \text {. }
$$


[This also defines what we mean by $\mathrm{U}(1)_{X}$.] Breaking the SU(5) symmetry to that of the standard model, we obtain

$$
\begin{aligned}
& \mathbf{1 0} \rightarrow(\mathbf{3}, \mathbf{2})_{-1,1 / 3}+(\overline{\mathbf{3}}, \mathbf{1})_{-1,-4 / 3}+(\mathbf{1}, \mathbf{1})_{-1,2}, \\
& \overline{\mathbf{5}} \rightarrow(\overline{\mathbf{3}}, \mathbf{1})_{3,2 / 3}+(\mathbf{1}, \mathbf{2})_{3,-1}, \quad \mathbf{1} \rightarrow(\mathbf{1}, \mathbf{1})_{-5,0},
\end{aligned}
$$

where the first and second subscript denotes the $\mathrm{U}(1)_{X}$ and $\mathrm{U}(1)_{Y}$ charge, respectively. In terms of Dynkin diagrams, this reads:

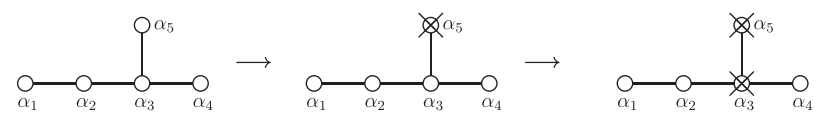

The direction corresponding to $\mathrm{U}(1)_{X}$ is given by:

$$
\begin{aligned}
\mathrm{Y}_{X} & =4 \alpha_{5}^{*}=4 \sum_{j=1}^{5}\left(A_{\mathrm{SO}(10)}^{-1}\right)_{5 j} \alpha_{j} \\
& =\left(2 \alpha_{1}+4 \alpha_{2}+6 \alpha_{3}+3 \alpha_{4}+5 \alpha_{5}\right)
\end{aligned}
$$

In flipped $\mathrm{SU}(5)$, hypercharge is a linear combination of this direction and B-L, namely:

$$
\mathrm{Y}_{\mathrm{fl}}=-\frac{1}{2} \mathrm{Y}_{X}-\frac{1}{2} \mathrm{Y}_{\mathrm{B}-\mathrm{L}}
$$

Calculating the hypercharge assignments for the elementary particles, it turns out that in comparison to Georgi-Glashow $\mathrm{SU}(5)$, the roles of $\bar{u}, \bar{d}$ and of $\bar{e}, \bar{\nu}$ are interchanged, cf. Table III.

\section{APPENDIX D: GEORGI-GLASHOW HYPERCHARGE IN DISGUISE}

Consider the following two models below, to which we will refer as

First Model

$$
\begin{aligned}
V_{6} & =\left(\begin{array}{lllllllllllllllll}
\frac{1}{3} & \frac{1}{2} & -\frac{1}{3} & -\frac{1}{6} & -\frac{1}{6} & -\frac{1}{6} & -\frac{1}{6} & -\frac{1}{6} & \frac{1}{12} & \frac{1}{4} & \frac{1}{12} & -\frac{1}{12} & -\frac{1}{12} & -\frac{1}{12} & \frac{11}{12} & \frac{11}{12}
\end{array}\right) \\
W_{3} & =\left(\begin{array}{llllllllllllllllll}
-\frac{1}{6} & \frac{1}{2} & \frac{1}{6} & -\frac{1}{6} & -\frac{1}{6} & \frac{1}{6} & \frac{7}{6} & \frac{7}{6} & \frac{7}{6} & -\frac{5}{6} & -\frac{5}{6} & -\frac{5}{6} & -\frac{5}{6} & -\frac{5}{6} & -\frac{25}{6} & -\frac{13}{6}
\end{array}\right) \\
W_{2} & =\left(\begin{array}{llllllllllllllllll}
-\frac{3}{4} & -\frac{1}{4} & -\frac{1}{4} & -\frac{1}{4} & -\frac{1}{4} & \frac{1}{4} & \frac{5}{4} & \frac{5}{4} & \frac{3}{4} & \frac{1}{4} & -\frac{1}{4} & -\frac{3}{4} & -\frac{3}{4} & -\frac{3}{4} & \frac{25}{4} & \frac{29}{4}
\end{array}\right)
\end{aligned}
$$

Second Model

$$
\begin{aligned}
V_{6} & =\left(\begin{array}{llllllllllllllllll}
\frac{2}{3} & \frac{1}{3} & \frac{1}{6} & \frac{1}{6} & 0 & 0 & 0 & 0 & -\frac{1}{6} & \frac{1}{2} & \frac{1}{2} & \frac{1}{2} & \frac{1}{2} & \frac{1}{2} & -\frac{1}{2} & \frac{1}{2}
\end{array}\right) \\
W_{3} & =\left(\begin{array}{llllllllllllllll}
\frac{1}{6} & -\frac{1}{6} & \frac{1}{6} & \frac{1}{6} & -\frac{1}{2} & \frac{1}{6} & \frac{1}{6} & -\frac{1}{6} & -\frac{1}{3} & \frac{2}{3} & \frac{2}{3} & \frac{1}{3} & \frac{1}{3} & \frac{1}{3} & \frac{1}{3} & -\frac{1}{3}
\end{array}\right) \\
W_{2} & =\left(\begin{array}{lllllllllllllllll}
-\frac{3}{4} & \frac{1}{4} & \frac{3}{4} & -\frac{3}{4} & -\frac{1}{4} & -\frac{1}{4} & -\frac{1}{4} & \frac{1}{4} & -\frac{1}{4} & -\frac{1}{4} & -\frac{1}{4} & -\frac{3}{4} & -\frac{3}{4} & -\frac{3}{4} & \frac{1}{4} & -\frac{1}{4}
\end{array}\right)
\end{aligned}
$$

for the sake of brevity. The gauge group in both cases is

$$
\mathrm{SU}(3) \times \mathrm{SU}(2) \times \mathrm{SU}(2) \times \mathrm{U}(1)^{4} \times \mathrm{SU}(7)^{\prime} \times \mathrm{U}(1)^{\prime 2},
$$

and their spectra coincide:

\begin{tabular}{lllll}
\hline \hline $3 \times(\mathbf{3}, \mathbf{2}, \mathbf{1}, \mathbf{1})$ & $3 \times(\overline{\mathbf{3}}, \mathbf{1}, \mathbf{2}, \mathbf{1})$ & $2 \times(\mathbf{1}, \mathbf{2}, \mathbf{2}, \mathbf{1})$ & $4 \times(\mathbf{1}, \mathbf{1}, \mathbf{1}, \mathbf{7})$ & $34 \times(\mathbf{1}, \mathbf{1}, \mathbf{1}, \mathbf{1})$ \\
\hline & $12 \times(\mathbf{3}, \mathbf{1}, \mathbf{1}, \mathbf{1})$ & $13 \times(\mathbf{1}, \mathbf{2}, \mathbf{1}, \mathbf{1})$ & $4 \times(\mathbf{1}, \mathbf{1}, \mathbf{1}, \overline{\mathbf{7}})$ & \\
& $12 \times(\overline{\mathbf{3}}, \mathbf{1}, \mathbf{1}, \mathbf{1})$ & $17 \times(\mathbf{1}, \mathbf{1}, \mathbf{2}, \mathbf{1})$ & & \\
\hline \hline
\end{tabular}

In both cases, the shift $V_{6}$ breaks

$$
\mathrm{E}_{8} \rightarrow \mathrm{SO}(10) \times \mathrm{SU}(2) \times \mathrm{SU}(2) .
$$

For the first model, the Wilson lines break to:

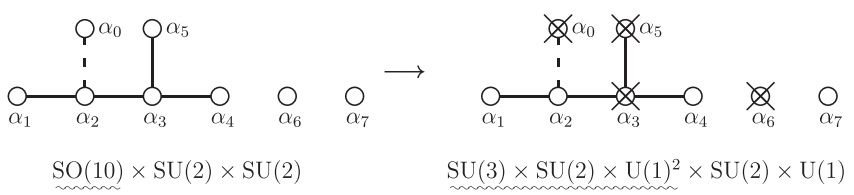

The underlining indicates the breaking of $\mathrm{SO}(10)$.
In contrast, in the second model, the Wilson lines break to:

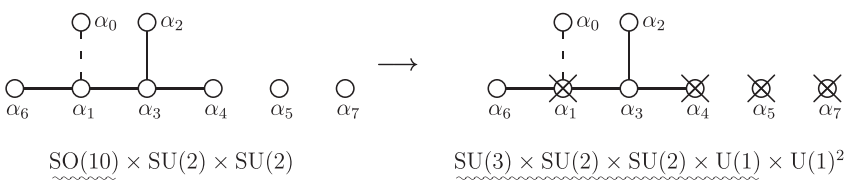

In both cases, the resulting gauge group and the spectra are the same. The difference lies in the way how the $\mathrm{SO}(10)$ breaks. For the first model, hypercharge that lies in $\mathrm{SO}(10)$ 
(see Appendix C) gives a vectorlike spectrum, whereas for the second model, we cannot even attempt to construct this hypercharge direction, since the intermediate $\mathrm{SO}(10)$ is seemingly missing. Our general ansatz will yield a hypercharge direction in either case, but for the second model, the connection between the underlying $\mathrm{SO}(10)$ and hypercharge is disguised. This complication is not relevant for the mini-landscape search, since there, we first constructed the hypercharge direction and then identified the models with identical non-Abelian spectra.

\section{APPENDIX E: GEORGI-GLASHOW UNIFICATION WITHOUT SU(5)}

Consider the following model with shift and Wilson lines

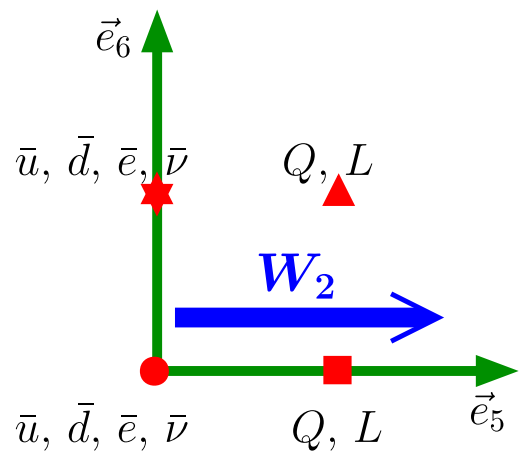

FIG. 7 (color online). Localization of the matter fields in the 3rd torus.

$$
\begin{aligned}
V_{6} & =\left(\begin{array}{lllllllllllllllll}
\frac{2}{3} & \frac{1}{3} & \frac{1}{6} & \frac{1}{6} & 0 & 0 & 0 & 0 & \frac{1}{3} & 0 & 0 & 0 & 0 & 0 & 0 & 0
\end{array}\right) \\
W_{3} & =\left(\begin{array}{llllllllllllllll}
\frac{1}{6} & \frac{1}{6} & -\frac{1}{6} & -\frac{1}{2} & \frac{1}{2} & \frac{1}{6} & \frac{1}{6} & \frac{1}{6} & -\frac{2}{3} & \frac{1}{3} & \frac{1}{3} & 0 & 0 & 0 & 0 & 0
\end{array}\right) \\
W_{2} & =\left(\begin{array}{llllllllllllllll}
-\frac{1}{4} & \frac{1}{4} & \frac{1}{4} & \frac{1}{4} & \frac{1}{4} & -\frac{1}{4} & -\frac{1}{4} & -\frac{1}{4} & 0 & -\frac{1}{2} & \frac{1}{2} & 0 & 0 & 0 & 0 & 0
\end{array}\right)
\end{aligned}
$$

and hypercharge

$$
Y=\left(\begin{array}{llllllllllllllll}
\frac{1}{2} & -\frac{1}{2} & -\frac{1}{2} & -\frac{1}{2} & \frac{3}{2} & -\frac{1}{6} & -\frac{1}{6} & -\frac{1}{6} & 0 & 0 & 0 & 0 & 0 & 0 & 0 & 0
\end{array}\right) .
$$

The shift and Wilson lines break

$$
\begin{aligned}
\mathrm{E}_{8} & \times \mathrm{E}_{8}^{\prime} \stackrel{V}{\rightarrow} \mathrm{SO}(10) \times \mathrm{SU}(2) \times \mathrm{SU}(2) \times \mathrm{SO}(14)^{\prime} \stackrel{W_{3}}{\rightarrow} \mathrm{SU}(4) \\
& \times \mathrm{SO}(10)^{\prime} \times \mathrm{SU}(2)^{\prime} \stackrel{W_{2}}{\rightarrow} \mathrm{SU}(3) \times \mathrm{SO}(10)^{\prime} \times \mathrm{SU}(2)^{\prime} .
\end{aligned}
$$

The gauge groups for the color and weak interactions are located in the first and second $E_{8}$, respectively, whereas hypercharge lies fully in the first one. As such, the matter representations like $(3,2)$ cannot originate from the $\mathbf{1 6}$ of $\mathrm{SO}(10)$, since $\mathrm{SU}(3) \times \mathrm{SU}(2) \not \subset \mathrm{SO}(10)$ in the first place.

Nevertheless, evaluating $Y$ on the $(\mathbf{1 6}, \mathbf{1}, \mathbf{1}, \mathbf{1})$ which lives at the origin (for $W_{3}=W_{2}=0$ ), we recover the standard charges. A quick calculation shows that $Y$ lies in $\mathrm{SO}(10)$, so it corresponds to either regular or flipped $\mathrm{SU}(5)$ for some choice of simple roots of $\mathrm{SO}(10)$.

It is still true that the $\mathbf{1 6}$ is unaffected by the orbifold projections, so the massless states which we expect to survive are

$$
\begin{aligned}
\mathbf{1 6} \rightarrow & (\mathbf{3}, \mathbf{1})_{1 / 3}+(\mathbf{3}, \mathbf{1})_{1 / 3}+(\overline{\mathbf{3}}, \mathbf{1})_{-4 / 3}+(\overline{\mathbf{3}}, \mathbf{1})_{2 / 3} \\
& +(\mathbf{1}, \mathbf{1})_{-1}+(\mathbf{1}, \mathbf{1})_{-1}+(\mathbf{1}, \mathbf{1})_{2}+(\mathbf{1}, \mathbf{1})_{0}
\end{aligned}
$$

at the origin in the 5th twisted sector, and a quick check shows that this is indeed true. The big difference is that only $\bar{u}, \bar{d}, \bar{e}$ and $\bar{\nu}$ (see Table I) live in this $\mathbf{1 6}$ at the origin, and the other representations in the multiplet have an interpretation as exotic particles. Since there is no Wilson line along the $\vec{e}_{6}$ in the torus, the spectrum is degenerate along this direction.

In Fig. 7, we present the localization of the standard model fields. There are $2 \times(\mathbf{3}, \mathbf{2})_{1 / 3}$ in the 5 th twisted sector living at $\boldsymbol{\Delta}$ and $\boldsymbol{a}$, respectively. Their highest weights are

$$
\begin{aligned}
& Q_{\mathbf{\Lambda}}=\left(\begin{array}{llllllllllllllll}
\frac{1}{12} & -\frac{1}{12} & \frac{1}{12} & \frac{1}{12} & \frac{1}{4} & \frac{3}{4} & -\frac{1}{4} & -\frac{1}{4} & -\frac{1}{3} & \frac{1}{2} & -\frac{1}{2} & 0 & 0 & 0 & 0 & 0
\end{array}\right) \text {, } \\
& Q_{\mathbf{\square}}=\left(\begin{array}{llllllllllllllll}
\frac{1}{12} & -\frac{1}{12} & \frac{1}{12} & \frac{1}{12} & \frac{1}{4} & \frac{3}{4} & -\frac{1}{4} & -\frac{1}{4} & -\frac{1}{3} & \frac{1}{2} & -\frac{1}{2} & 0 & 0 & 0 & 0 & 0
\end{array}\right) \text {. }
\end{aligned}
$$

These states are not part of a $\mathbf{1 6}$ or any other $\mathrm{SO}(10)$ irreducible representation, as can be seen by calculating the Dynkin labels w.r.t. the simple roots. Nevertheless, the scalar product with $Y$ gives the correct hypercharge for a left-handed quark doublet in both cases. 
[1] O. Lebedev et al., Phys. Lett. B 645, 88 (2007).

[2] T. Kobayashi, S. Raby, and R.-J. Zhang, Phys. Lett. B 593, 262 (2004).

[3] S. Förste, H.P. Nilles, P. K.S. Vaudrevange, and A. Wingerter, Phys. Rev. D 70, 106008 (2004).

[4] T. Kobayashi, S. Raby, and R.-J. Zhang, Nucl. Phys. B704, 3 (2005).

[5] W. Buchmüller, K. Hamaguchi, O. Lebedev, and M. Ratz, arXiv:hep-th/0606187.

[6] W. Buchmüller, K. Hamaguchi, O. Lebedev, and M. Ratz, Nucl. Phys. B712, 139 (2005).

[7] W. Buchmüller, K. Hamaguchi, O. Lebedev, and M. Ratz, Phys. Rev. Lett. 96, 121602 (2006).

[8] W. Buchmüller, K. Hamaguchi, O. Lebedev, and M. Ratz, arXiv:hep-ph/0512326.

[9] S. Förste, H. P. Nilles, and A. Wingerter, Phys. Rev. D 72, 026001 (2005).

[10] S. Förste, H. P. Nilles, and A. Wingerter, Phys. Rev. D 73, 066011 (2006).

[11] H. Georgi and S. L. Glashow, Phys. Rev. Lett. 32, 438 (1974).

[12] J. C. Pati and A. Salam, Phys. Rev. D 10, 275 (1974).

[13] H. Fritzsch and P. Minkowski, Ann. Phys. (N.Y.) 93, 193 (1975).

[14] R. Slansky, Phys. Rep. 79, 1 (1981).

[15] T. Asaka, W. Buchmüller, and L. Covi, Phys. Lett. B 523, 199 (2001).

[16] S. Dimopoulos, S. A. Raby, and F. Wilczek, Phys. Today 44N10, 25 (1991).

[17] H. P. Nilles, arXiv:hep-th/0410160.

[18] H. Esmaeili, N. Mahdavi-Amiri, and E. E. Spedicato, Numer. Math. 90, 101 (2001).

[19] A. K. Lenstra, H. W. Lenstra, and L. Lovász, Math. Ann. 261, 515 (1982).
[20] V. Shoup, http://shoup.net/ntl.

[21] B. Buchberger, Ph.D. thesis, Universitaet Innsbruck, 1965.

[22] G.-M. Greuel, G. Pfister, and H. Schönemann, http:// www.singular.uni-kl.de.

[23] P. Di Francesco, P. Mathieu, and D. Senechal, Conformal Field Theory (Springer, New York, USA, 1997).

[24] Numerical Algorithms Group, (http://www.nag.co.uk).

[25] O. Lebedev, H.-P. Nilles, S. Raby, S. Ramos-Sanchez, M. Ratz, P. Vaudrevange, and A. Wingerter (unpublished).

[26] K. R. Dienes, A. E. Faraggi, and J. March-Russell, Nucl. Phys. B467, 44 (1996).

[27] L. J. Hall and Y. Nomura, Phys. Rev. D 64, 055003 (2001).

[28] R. Contino, L. Pilo, R. Rattazzi, and E. Trincherini, Nucl. Phys. B622, 227 (2002).

[29] K. R. Dienes, E. Dudas, and T. Gherghetta, Phys. Lett. B 436, 55 (1998).

[30] H. D. Kim and S. Raby, J. High Energy Phys. 01 (2003) 056.

[31] A. Hebecker and M. Trapletti, Nucl. Phys. B713, 173 (2005).

[32] L. E. Ibanez and D. Lust, Nucl. Phys. B382, 305 (1992).

[33] J. Giedt, Ann. Phys. (N.Y.) 297, 67 (2002).

[34] C. Bauer, A. Frink, and R. Kreckel, arXiv:cs.sc/0004015.

[35] W. Adams and P. Loustaunau, in An Introduction to Gröbner Bases (AMS, Providence, RI, 1996).

[36] T. Becker and V. Weisspfenning, Gröbner Bases - A Computational Approach to Commutative Algebra (Springer, New York, 1993).

[37] J. Gray, Y.-H. He, and A. Lukas, J. High Energy Phys. 09 (2006) 031.

[38] M.B. Green, J.H. Schwarz, and E. Witten, Superstring Theory. Vol. 1: Introduction (University Press, Cambridge, England, 1987). 